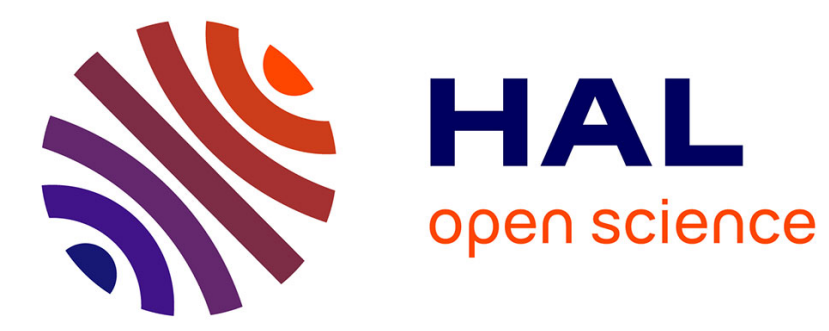

\title{
Structure and dynamics of FCHo2 docking on membranes
}

F. El El Alaoui, I. Casuso, D. Sanchez-Fuentes, C. André-Arpin, R. Rathar, V. Baecker, A. Castro, T. Lorca, J. Viaud, S. Vassilopoulos, et al.

\section{- To cite this version:}

F. El El Alaoui, I. Casuso, D. Sanchez-Fuentes, C. André-Arpin, R. Rathar, et al.. Structure and dynamics of FCHo2 docking on membranes. 2021. hal-03437156

\section{HAL Id: hal-03437156 https://hal.science/hal-03437156}

Preprint submitted on 19 Nov 2021

HAL is a multi-disciplinary open access archive for the deposit and dissemination of scientific research documents, whether they are published or not. The documents may come from teaching and research institutions in France or abroad, or from public or private research centers.
L'archive ouverte pluridisciplinaire HAL, est destinée au dépôt et à la diffusion de documents scientifiques de niveau recherche, publiés ou non, émanant des établissements d'enseignement et de recherche français ou étrangers, des laboratoires publics ou privés. 


\title{
Structure and dynamics of FCHo2 docking on membranes
}

\author{
F. El Alaoui ${ }^{1}$, I. Casuso², D. Sanchez-Fuentes ${ }^{3}$, C. André-Arpin ${ }^{1}$, R. Rathar ${ }^{3}$, V. Baecker ${ }^{4}$, A. Castro ${ }^{5}$, \\ T. Lorca ${ }^{5}$, J. Viaud ${ }^{6}$, S. Vassilopoulos ${ }^{7}$, A. Carretero-Genevrier ${ }^{3}$, L. Picas ${ }^{1, *}$.
}

${ }^{1}$ Institut de Recherche en Infectiologie de Montpellier (IRIM). CNRS UMR 9004 - Université de Montpellier, Montpellier, France.

${ }^{2}$ U1067 INSERM, Aix-Marseille Université, Marseille, France

${ }^{3}$ Institut d'Électronique et des Systèmes (IES), CNRS UMR 5214 - Université de Montpellier, Montpellier, France.

${ }^{4}$ Montpellier Ressources Imagerie, BioCampus Montpellier, CNRS, INSERM, University of Montpellier, 34000 Montpellier, France.

${ }^{5}$ Centre de Biologie Cellulaire de Montpellier (CRBM). CNRS UMR - Université de Montpellier, Montpellier, France.

${ }^{6}$ Institute of Metabolic and Cardiovascular Diseases (I2MC), UMR1297, Toulouse, France; University of Toulouse, Paul Sabatier University, Toulouse, France.

${ }^{7}$ Sorbonne Université, INSERM, Institute of Myology, Centre of Research in Myology, UMRS 974, Paris, France.

*Correspondence sent to: laura.picas@ @irim.cnrs.fr

\begin{abstract}
Clathrin-mediated endocytosis (CME) is a central trafficking pathway in eukaryotic cells regulated by phosphoinositides. The plasma membrane phosphatidylinositol-4,5-bisphosphate $\left(\mathrm{PI}(4,5) \mathrm{P}_{2}\right)$ plays an instrumental role in driving CME initiation. The F-BAR domain only protein 1 and 2 complex (FCHo1/2) is among the early proteins that reach the plasma membrane, but the exact mechanisms triggering its recruitment remains elusive. Here, we show the molecular dynamics of FCHo2 selfassembly on membranes by combining bottom-up synthetic approaches on in vitro and cellular membranes. Our results indicate that $\mathrm{PI}(4,5) \mathrm{P}_{2}$ domains assist $\mathrm{FCHo} 2$ docking at specific membrane regions, where it self-assembles into ring-like shape protein patches. We show that binding of FCHo2 on cellular membranes promotes $\mathrm{PI}(4,5) \mathrm{P}_{2}$ clustering at the boundary of cargo receptors and that this accumulation enhances clathrin assembly. Thus, our results provide a mechanistic framework that could explain the recruitment of early $\mathrm{PI}(4,5) \mathrm{P}_{2}$-interacting proteins at endocytic sites.
\end{abstract}




\section{Introduction}

The biogenesis of clathrin-coated vesicles requires precise and coordinated recruitment of more than 50 different proteins to undergo the bending, elongation, and fission of the plasma membrane $(1,2)$. Different factors assist in recruiting endocytic proteins, such as the interaction with phosphoinositides(3), curvature sensing, and protein-protein interactions(4). Also, the membrane tension that results from membrane-cytoskeleton adhesion sets the load of forming transport vesicles(5). Although the initiation of endocytosis is a critical step, the exact mechanism triggering the nucleation of endocytic proteins at the plasma membrane is not well understood. The early stages of CME entail the nucleation of adaptor and accessory proteins, cargo, and lipids to undergo the bending of the plasma membrane(6). Several studies have shown that FCHo1/2 (Fer/CIP4 homology domain only protein 1 or 2$)$ is among the early proteins recruited at endocytic sites $(1,7)$, where it establishes a network of interactions with pioneer proteins, such as Eps15, adaptor protein 2 (AP2), and transmembrane cargo(1, 8, 9). FCHo paralogs associate with membranes through the dimerization of F-BAR domains displaying a shallow concave surface that interacts with acidic phospholipids $(7,10,11)$. A polybasic motif follows the F-BAR scaffold and provides a selective recognition for $\mathrm{PI}(4,5) \mathrm{P}_{2}(8)$. Finally, FCHo1/2 is flanked at the C-terminal by a $\mu$-homology domain $(\mu-\mathrm{HD})$ that directly binds with multiple early endocytic proteins such as Eps15, intersectin 1 or $\operatorname{CALM}(7,8,12)$. Indeed, FCHo1/2 is required to recruit Eps15 on membranes(13), and the assembly of a FCHo1/2-Eps15-AP2 complex is essential to drive efficient cargo loading(8). The recruitment of FCHo1/2 on membranes is central to initiate the endocytic activity but, the underlying molecular mechanism remains unclear.

Here we combined sub-diffraction microscopy and high-speed atomic force microscopy (HS-AFM) with in vitro and in cellulo systems to show that $\mathrm{PI}(4,5) \mathrm{P}_{2}$ domains regulate $\mathrm{FCHo} 2$ docking on flat membranes, where it self-assembles into ring-like shaped protein structures that are compatible with the size and temporal scale of clathrin-mediated endocytosis. Our results indicate that, in the absence of metabolizing enzymes, FCHo2 can engage a local $\mathrm{PI}(4,5) \mathrm{P}_{2}$ enrichment at the boundaries of clathrinregulated cargo receptors and enhance the formation of clathrin-positive assemblies. Finally, manipulation of membrane curvature through lithographic approaches showed that $\mathrm{PI}(4,5) \mathrm{P}_{2}$ promotes the partition of $\mathrm{FCHo} 2$ at the edges of dome-like structures. Collectively, our work points out $\mathrm{PI}(4,5) \mathrm{P}_{2}$ lateral lipid heterogeneities as an organizing mechanism supporting the docking and self-organization of $\mathrm{PI}(4,5) \mathrm{P}_{2}$-interacting proteins that, like $\mathrm{FCHo}$, participate in the initial stages of CME.

\section{Results}

\section{PI $(4,5) P_{2}$ domains drive FCHo2 docking on membranes}


To study FCHo1/2 recruitment on cellular membranes, we monitored by airyscan microscopy the binding of recombinant full-length FCHo2-Alexa647 on plasma membrane sheets. We generated plasma membrane sheets by ultrasound-mediated unroofing of cells stably expressing the transferrin receptor (TfR-GFP) as a model of cargo receptor regulated by FCHo1/2(7). To monitor the subcellular dynamics of lipids we used TopFluor fatty acid conjugates, as previously reported(14). We loaded HT1080 unroofed cells with TF-TMR-PI(4,5) $\mathrm{P}_{2}$ or fluorescent phosphatidylserine as a control (PS, TF-TMRPS) (Figure 1A and B). The functionality of recombinant FCHo2 was determined by performing a tubulation assay using membrane sheets made of brain polar lipids, as previously reported(15) (Figure S1). Kymograph analysis on plasma membrane sheets showed that $\mathrm{FCHo} 2$ is preferentially recruited to $\mathrm{PI}(4,5) \mathrm{P}_{2}$-enriched regions often co-localized with the TfR (Figure 1C). We found that on these regions the kinetics of FCHo2 recruitment was faster than in the absence of $\mathrm{PI}(4,5) \mathrm{P}_{2}$ enrichment (Figure 1D). Indeed, the appearance of $\mathrm{FCHo} 2$ puncta on plasma membrane sheets showed a positive correlation with the intensity of $\mathrm{PI}(4,5) \mathrm{P}_{2}$ compared to PS (Figure 1E).

To determine if the spatial recruitment of $\mathrm{FCHo} 2$ is mediated by $\mathrm{PI}(4,5) \mathrm{P}_{2}$-enriched domains, we first estimated the protein binding on supported lipid bilayers made of $20 \%$ of total negatively charged lipids and different $\% \mathrm{~mol}$ of $\mathrm{PI}(4,5) \mathrm{P}_{2}$. As expected, increasing amounts of $\mathrm{PI}(4,5) \mathrm{P}_{2}$ favored $\mathrm{FCHo} 2$ binding on flat membranes (Figure 1F). Next, we analyzed the spatial recruitment of FCHo2 on 5\% PI(4,5) $\mathrm{P}_{2^{-}}$ containing supported lipid bilayers doped with $0.2 \%$ mol of fluorescent PI(4,5)P2 (TF-TMR-PI(4,5) $\mathrm{P}_{2}$ ) and $0.2 \%$ of Oregon Green 488 1,2-Dihexadecanoyl-sn-Glycero-3-Phosphoethanolamine (OG-DHPE) at the expenses of their non-labeled counterpart (Figure S2). As a result, we designed as PI(4,5) $\mathrm{P}_{2-}$ enriched domains the existence of spots that displayed a local increase in the TF-TMR-PI $(4,5) \mathrm{P}_{2}$ signal along with a decrease in the intensity of OG-DHPE, as compared to a homogeneous distribution of lipids characterized by an equivalent partitioning of both lipid dyes (Figure S2). We characterized the steadystate organization of the fluorescent $\mathrm{PI}(4,5) \mathrm{P}_{2}$ into small domains as compared to other anionic lipids (Figure S3), in agreement with previous numerical simulations(16). The temporal analysis of FCHo2 recruitment (Figure $1 \mathrm{G}$ ) showed that binding to $\mathrm{PI}(4,5) \mathrm{P}_{2}$ domains resulted in the formation of longlived FCHo2 puncta (red arrow, Figure 1H), whereas its association with a homogenous distribution of $\mathrm{PI}(4,5) \mathrm{P}_{2}$ was more likely to lead to FCHo2 disassembly (gray arrow, Figure 1H).

Next, we analyzed the contribution of the F-BAR domain on the preferential localization of the fulllength protein on $\mathrm{PI}(4,5) \mathrm{P}_{2}$-domains (Figure 1I). Our results show that the binding of both proteins on membranes follows a positive correlation with the existence of $\mathrm{PI}(4,5) \mathrm{P}_{2}$ domains. Although this correlation was more significant for $\mathrm{FCHo} 2$, the preferential recruitment of the F-BAR domain to $\mathrm{PI}(4,5) \mathrm{P}_{2}$ enriched regions is in agreement with the enhanced binding of the domain at $5 \%$ mol of $\mathrm{PI}(4,5) \mathrm{P}_{2}$ (Figure $\mathrm{S} 4$ ). Collectively, these results indicate that $\mathrm{PI}(4,5) \mathrm{P}_{2}$-enriched regions act as docking 
sites for FCHo2 recruitment on membranes through the electrostatic interaction of the F-BAR and polybasic motif.

\section{A}
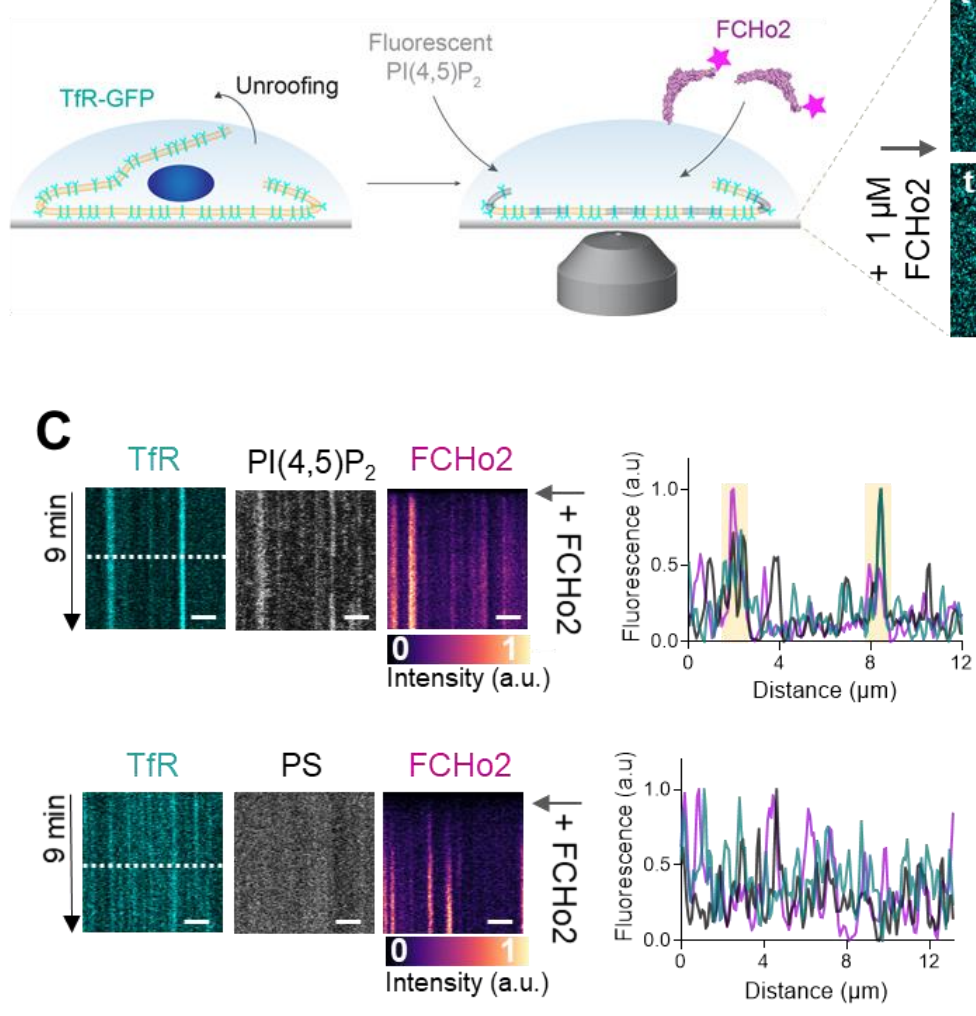

E
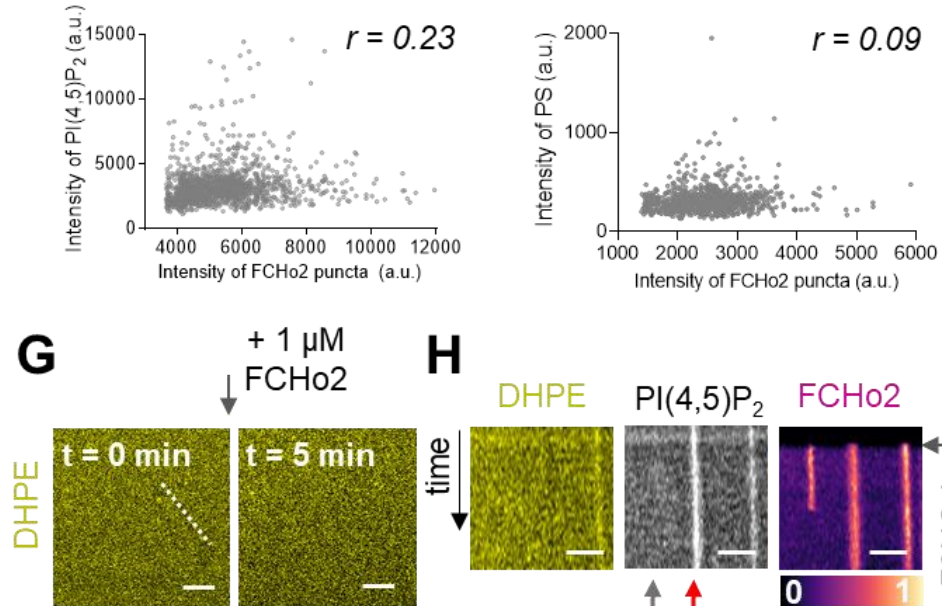

$+1 \mu \mathrm{M}$

FCHo2
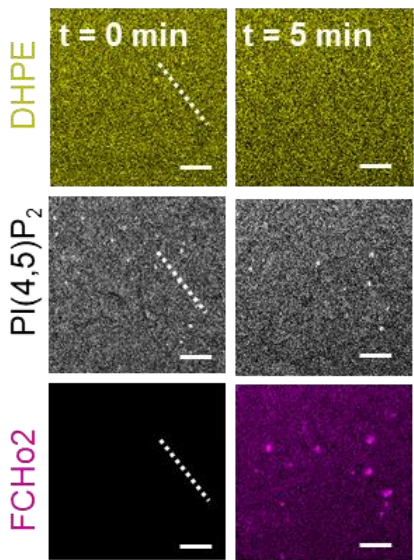

H

DHPE $\quad \mathrm{PI}(4,5) \mathrm{P}_{2} \quad \mathrm{FCHO} 2$
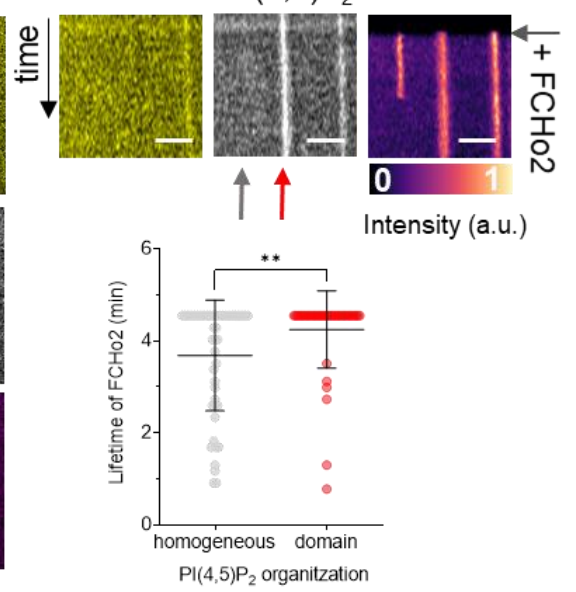

B

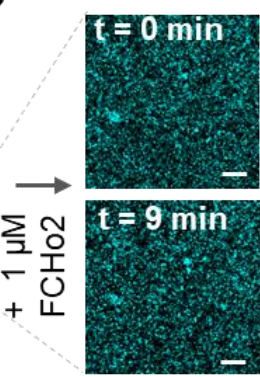

$\mathrm{PI}(4,5) \mathrm{P}_{2}$

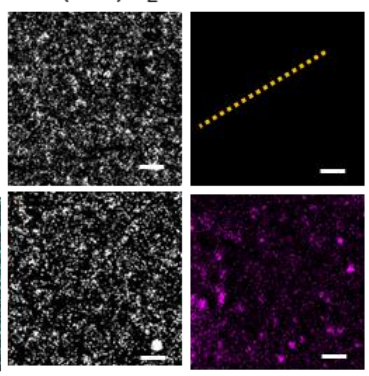

On $\mathrm{PI}(4,5) \mathrm{P}_{2}$
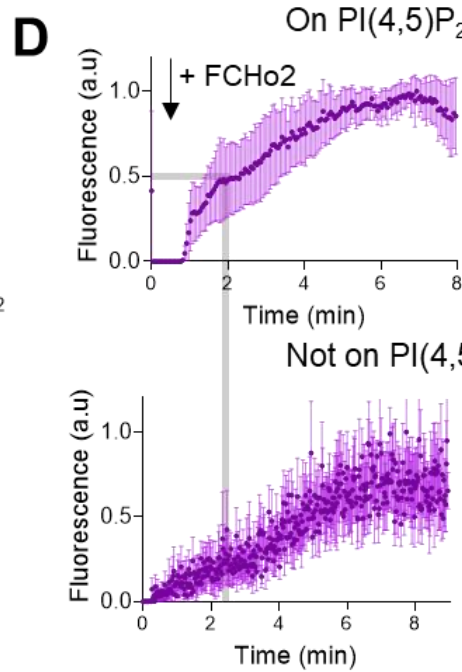

$\mathbf{F}$
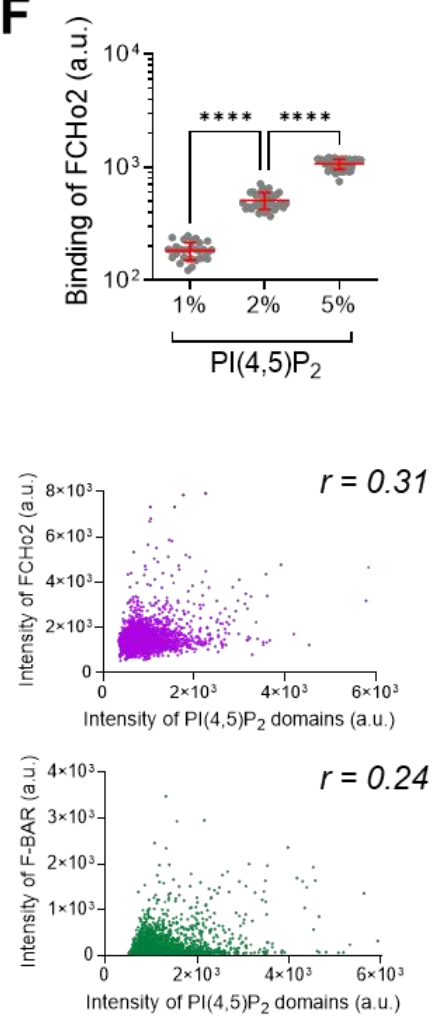
Figure 1. FCHo2 is recruited to $\mathrm{PI}(4,5) \mathrm{P}_{2}$-enriched regions on cellular and in vitro membranes. (A) Cartoon of the experimental setup to monitor the recruitment of FCHo2-Alexa647 on cellular membranes doped with fluorescent $\mathrm{PI}(4,5) \mathrm{P}_{2}$. (B) Representative airyscan time-lapse showing the dynamics of PI(4,5) $\mathrm{P}_{2}$ (gray), FCHo2 (magenta) and TfR-GFP (cyan) on plasma membrane sheets. Scale bar, $5 \mu \mathrm{m}$. (C) Top, kymograph analysis along the dashed line in B on plasma membrane sheets doped with TF-TMR-PI(4,5) $\mathrm{P}_{2}$ and intensity profile of the TfR (cyan), PI(4,5) $\mathrm{P}_{2}$ (gray) and FCHo2 (magenta) along the dashed line in the kymograph. Time scale is 9 min. Bottom. Representative kymograph analysis on plasma membrane sheets doped with TF-TMR-PS and intensity profile of the TfR (cyan), PS (gray) and FCHo2 (magenta) along the dashed line in the kymograph. Time scale is $9 \mathrm{~min}$. Scale bar, $2 \mu \mathrm{m}$. (D) Fluorescence quantification over time of the FCHo2 recruitment (magenta) on $\mathrm{PI}(4,5) \mathrm{P}_{2}$-enriched regions (top) and not on $\mathrm{PI}(4,5) \mathrm{P}_{2}$ (bottom) on plasma membrane sheets. Each curve represents the mean \pm s.d., $n=7$. (E) Scatter plots of the correlation between the intensity of FCHo2 puncta and $\mathrm{PI}(4,5) \mathrm{P}_{2}$ or PS intensity. $\mathrm{n}=11448$ and $\mathrm{n}=1090$, respectively. The correlation coefficient was $\mathrm{r}=0.23$ and $\mathrm{r}=0.09$, respectively. (F) Binding of FCHo2 on lipid membranes containing different $\%$ mol of $\mathrm{PI}(4,5) \mathrm{P}_{2}(20 \%$ of total negative charge). Mean \pm s.d. in red. one-way ANOVA (****, P < 0.0001). $\mathrm{n}=31, \mathrm{n}=39$, and $\mathrm{n}=54$ for $1 \%, 2 \%$, and $5 \% \mathrm{~mol}$ of $\mathrm{PI}(4,5) \mathrm{P}_{2}$, respectively. (G) Airyscan timelapse showing the dynamics of FCHo2 (magenta) on $5 \%$ of $\mathrm{PI}(4,5) \mathrm{P}_{2}$ membranes doped with fluorescent $\mathrm{PI}(4,5) \mathrm{P}_{2}$ (gray) and DHPE (yellow). Scale bar, $2 \mu \mathrm{m}$. (H) Top, kymograph analysis along the white dashed line in F. Time scale, 5 min. Scale, 2 $\mu \mathrm{m}$. Bottom, lifetime of FCHo2 (in min) relative to the $\mathrm{PI}(4,5) \mathrm{P}_{2}$ organization: homogeneous (grey) and on domains (red), as highlighted by the arrows in the corresponding kymograph image. Mean \pm s.d. in black. Welch's t-test (**, $\mathrm{P}=0.0098)$. $\mathrm{n}=50$, $\mathrm{n}=43$ for a homogeneous and on domains, respectively. (I) Scatter plots of the correlation between the intensity of FCHo2 (purple) and F-BAR (green) relative to the intensity of $\mathrm{PI}(4,5) \mathrm{P}_{2}$ domains on $5 \%$ mol PI(4,5) $\mathrm{P}_{2}-$ membranes. $\mathrm{n}=11448$ for FCHo2 and $n=12828$ for F-BAR. The correlation coefficient, $r$, was $r=0.31$ and $r=0.24$, respectively.

\section{FCHo2-mediated PI(4,5) $P_{2}$ clustering primes pre-endocytic events}

The formation of $\mathrm{PI}(4,5) \mathrm{P}_{2}$ clusters at the plasma membrane orchestrates the recruitment of $\mathrm{PI}(4,5) \mathrm{P}_{2^{-}}$ binding proteins via ionic-lipid protein interactions $(17,18)$. Several structural domains of endocytic proteins, including the F-BAR domain of Syp1, locally accumulate $\mathrm{PI}(4,5) \mathrm{P}_{2}$ on in vitro membranes(19, 20), and we showed that BIN1 recruits its downstream partner dynamin through this mechanism. We thus investigated the impact of $\mathrm{FCHo} 2$ on $\mathrm{PI}(4,5) \mathrm{P}_{2}$ clustering formation. Injection of FCHo2-Alexa647 on TfR-GFP plasma membrane sheets led to the binding of the protein and the formation of submicrometric FCHo2-positive puncta that co-localized with $\mathrm{PI}(4,5) \mathrm{P}_{2}$ and the cargo receptor (Figure 2A). Analysis of FCHo2 dynamics showed that the protein binding convoyed an increase of the PI $(4,5) \mathrm{P}_{2}$ signal on TfR-GFP-positive puncta but not on PS labeled plasma membrane sheets. To determine if FCHo2-mediated $\mathrm{PI}(4,5) \mathrm{P}_{2}$ enrichment was a general feature of $\mathrm{FCHo}$, we monitored its binding relative another clathrin-regulated cargo, the EGF receptor (EGFR) (Figure 2B). In this case, we observed a concomitant increase of both the $\mathrm{PI}(4,5) \mathrm{P}_{2}$ and EGFR signal at FCHo2-postivie puncta (Figure 2B and S5), possibly as a result of the interaction of the EGFR juxta-membrane domain with $\mathrm{PI}(4,5) \mathrm{P}_{2}(21)$. Quantification of the intensity of $\mathrm{PI}(4,5) \mathrm{P}_{2}$ domains confirmed a local increase in the $\mathrm{PI}(4,5) \mathrm{P}_{2}$ signal after the addition of FCHo2 on cellular membranes (Figure 2C). Therefore, pointing out 
that membrane binding of $\mathrm{FCHo} 2$ induces $\mathrm{P}(4,5) \mathrm{P}_{2}$ clustering at the boundary of clathrin-regulated cargo receptors $(11,22)$.

We next analyzed if FCHo2-mediated $\mathrm{PI}(4,5) \mathrm{P}_{2}$ clustering participates in the formation of clathrincoated structures. To this end, we reconstituted in vitro clathrin-coat assembly by using cytosolic extracts, as previously reported $(23,24)$. We supplemented active cytosolic components from Xenopus egg extracts with a freshly prepared "energy mix", as detailed in the methods section. We determined by immunofluorescence the formation of clathrin-positive puncta on supported lipid bilayers containing $5 \%$ mol PI $(4,5) \mathrm{P}_{2}$ (including $0.2 \%$ of fluorescent $\left.\mathrm{PI}(4,5) \mathrm{P}_{2}\right)$ previously incubated with or without nonlabeled FCHo2 (Figure 2D). As expected, in the absence of FCHo2 the addition of cytosolic extracts leads to the appearance of clathrin-positive puncta, as compared to the energy mix alone (Figure $2 \mathrm{E}$ and S6). Under these conditions, we could also detect a residual signal of the FCHo1/2 antibody, corresponding to the endogenous protein present in the cytosolic extracts. Incubation of $5 \% \mathrm{PI}(4,5) \mathrm{P}_{2-}$ containing lipid bilayers with $1 \mu \mathrm{M}$ of FCHo2 resulted in a 1.7-fold increase in the mean intensity of clathrin-positive spots (Fig. 2E and F), which convoyed by a 3.6-fold local increase in the PI(4,5) $\mathrm{P}_{2^{-}}$ signal. We could detect a 2.5 -fold increase in the local $\mathrm{PI}(4,5) \mathrm{P}_{2}$ intensity in the presence of the F-BAR domain alone, which also lead to a moderate increase in the appearance of clathrin-positive spots, in agreement with the ability of the F-BAR domain in $\mathrm{PI}(4,5) \mathrm{P}_{2}$ clustering formation(19). Although FCHo2 was still able to bind on members, replacement of $\mathrm{PI}(4,5) \mathrm{P}_{2}$ by PS prevented the detection of clathrin in our in vitro assay, supporting the functional role of $\mathrm{PI}(4,5) \mathrm{P}_{2}$ in clathrin assembly on membranes. Thus, in addition to its membrane bending ability, these data indicate that $\mathrm{FCHo} 2$ might promote clathrin assembly by clustering $\mathrm{PI}(4,5) \mathrm{P}_{2}$. 
A

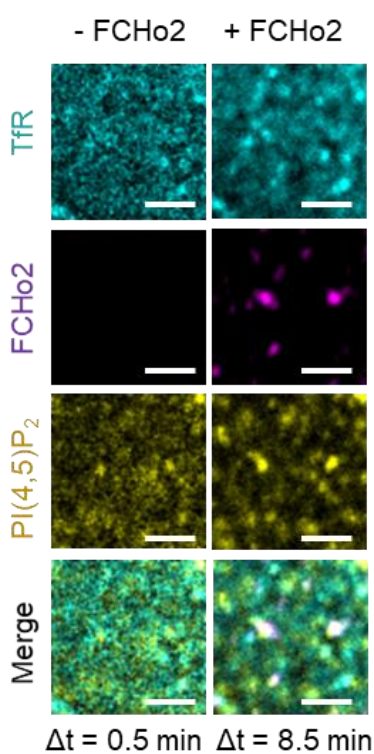

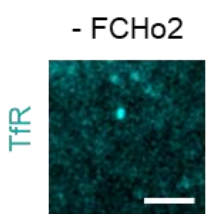
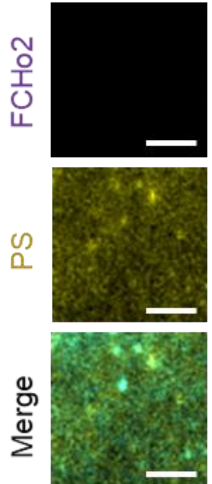

$\Delta \mathrm{t}=0.4 \mathrm{~min} \Delta \mathrm{t}=8.6 \mathrm{~min}$
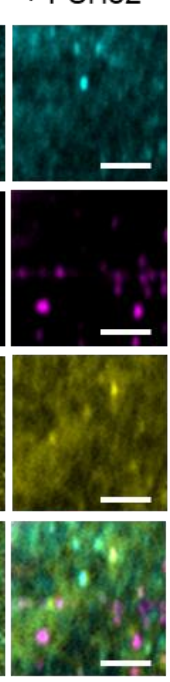

$\Delta \mathrm{t}=8.6 \mathrm{~min}$
B

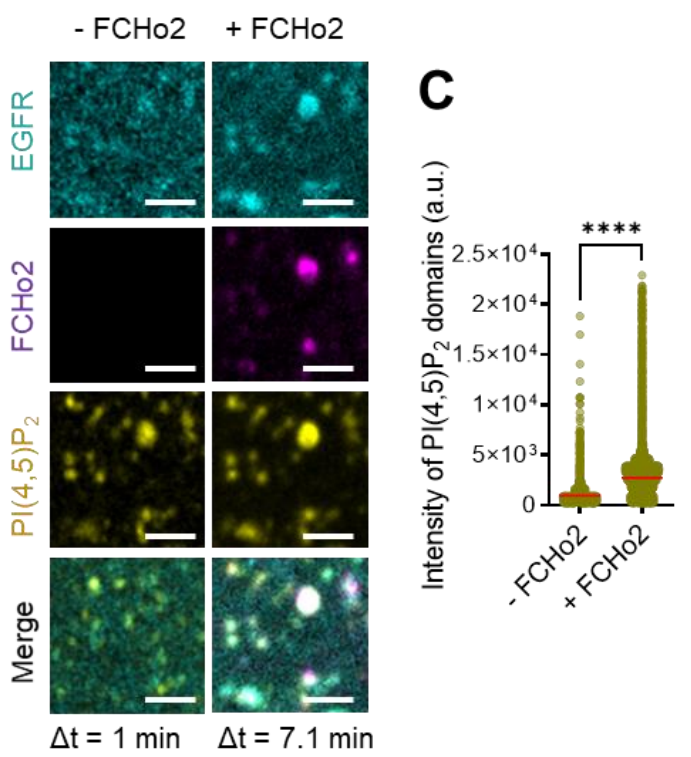

D

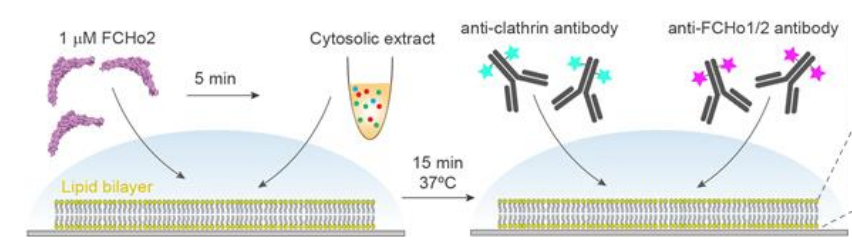

E
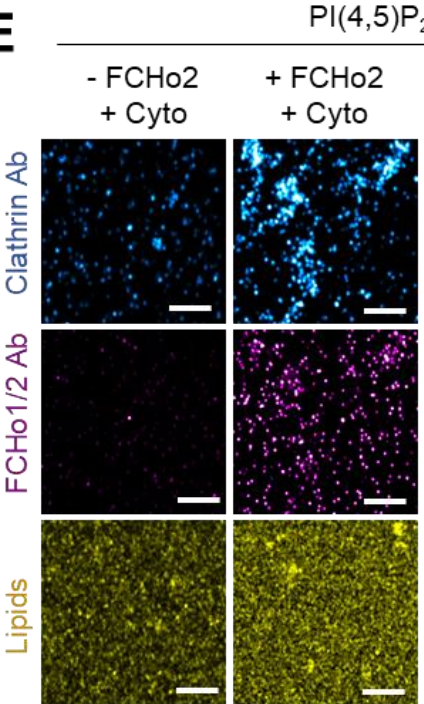

$\mathrm{PI}(4,5) \mathrm{P}_{2}$

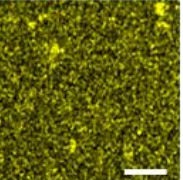


the co-localization with FCHo2 (magenta) and clathrin (cyan). Intensity profile along the orange dotted line in the corresponding image. Sale bar, $2 \mu \mathrm{m}$. (E) Representative airyscan images of lipid bilayers containing either 5\% mol PI(4,5) $\mathrm{P}_{2}$ or $20 \%$ PS (yellow) (total negative charge is $20 \%$ ) that were incubated with recombinant FCHo2 (+ FCHo2) or F-BAR domain (+ F-BAR) or without FCHo2 (- FCHo2) before addition of $4 \mathrm{mg} / \mathrm{ml}$ of cytosolic extracts (+ Cyto). Clathrin (blue) and FCHo1/2 (magenta) were detected by immunofluorescence (antibody, Ab). Sale bar, $2 \mu \mathrm{m}$. (F) Distribution of the intensity of clathrin structures in the presence or absence of $\mathrm{FCHo} 2$ or F-BAR on PI $(4,5) \mathrm{P}_{2}$ or PS-containing bilayers. Mean \pm s.d. is displayed in red and one-way ANOVA in black (****, $\mathrm{P}<0.0001$ ). $\mathrm{n}=7784, \mathrm{n}=7539, \mathrm{n}=4798$ and $\mathrm{n}=6642$, as in the graph. (G) Distribution of the intensity of fluorescent $\mathrm{PI}(4,5) \mathrm{P}_{2}$ on clathrin structures in the presence or absence of FCHo2 or F-BAR. Mean \pm s.d., in red. One-way ANOVA (****, $\mathrm{P}<0.0001) . \mathrm{n}=7784, \mathrm{n}=7539$ and $\mathrm{n}=6642$, as in the graph.

\section{Assembly of FCHo2 into molecular clusters prompts membrane bending}

A characteristic hallmark of endocytic proteins on cellular membranes is their spatial organization into punctate structures $(7,8)$, and we systematically observed this feature on plasma membrane sheets and in vitro membranes (Figure 1 and 2). Co-segregation of early endocytic proteins into sub-micrometer scale clusters relies on multivalent interactions(13). Therefore, we asked what might be the role of $\mathrm{PI}(4,5) \mathrm{P}_{2}$ in the spatial organization of $\mathrm{FCHo} 2$. We estimated by airyscan microscopy the average size of FCHo2 puncta on cellular membranes (i.e. plasma membrane sheets) and on 5\% $\mathrm{PI}(4,5) \mathrm{P}_{2}$ - containing bilayers after the addition of $1 \mu \mathrm{M}$ of FCHo2-Alexa 647 (Figure 3A and B), which was on both cases $0.067 \mu \mathrm{m}^{2}$. On lipid bilayers doped with $5 \% \mathrm{PI}(4,5) \mathrm{P}_{2}$ we could also detect that the F-BAR domain alone can assemble into punctate structures, although the average size was $\sim 0.094 \mu \mathrm{m}^{2}$. Interestingly, replacing $5 \% \mathrm{PI}(4,5) \mathrm{P}_{2}$ by PS lead to a homogeneous surface distribution of $\mathrm{FCHo} 2$ and prevented the detection of sub-micrometric puncta. Therefore, suggesting that $\mathrm{PI}(4,5) \mathrm{P}_{2}$ multivalent interactions through the F-BAR promote FCHo2 segregation into molecular clusters.

Next, we used atomic force microscopy (AFM) to investigate FCHo2 molecular clusters at nanometerscale resolution. Supported lipid bilayers doped with 5\% $\mathrm{PI}(4,5) \mathrm{P}_{2}$ were formed on freshly cleaved mica disks (see methods section). Before adding proteins, we confirmed the homogeneity and absence of defects of supported bilayers under the imaging buffer. Injection of full-length FCHo2 at $1 \mu \mathrm{M}$ in the imaging chamber resulted in sub-micrometric protein patches with a median dimension of $\sim 0.005 \mu \mathrm{m}^{2}$ that protruded out of the flat membrane surface with an average height of $\sim 47 \pm 3 \mathrm{~nm}$ (Figure 3C-E). An increase in the setpoint force from minimal values (a few tens of $\mathrm{pN}$ ) to intense forces (around one hundred $\mathrm{pN}$ ) resulted in the reduction of the height of FCHo2 clusters down to $\sim 15 \pm 2 \mathrm{~nm}$, thus suggesting that $\mathrm{FCHo} 2$ can moderately bend supported lipid membranes at minimal AFM imaging forces. 


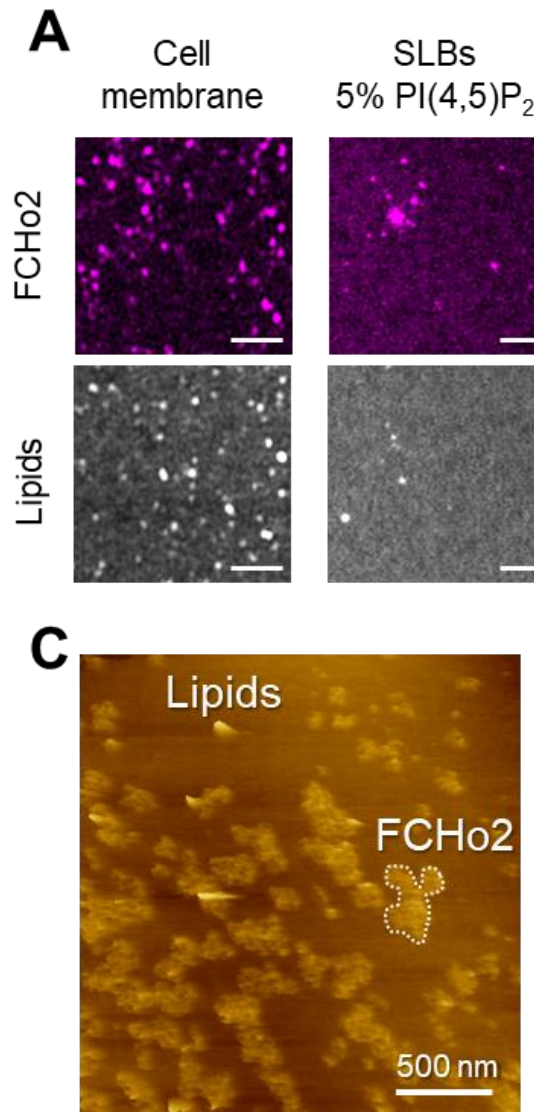

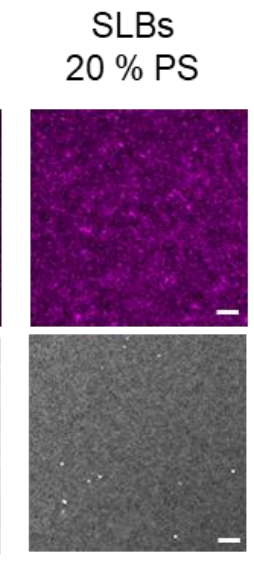
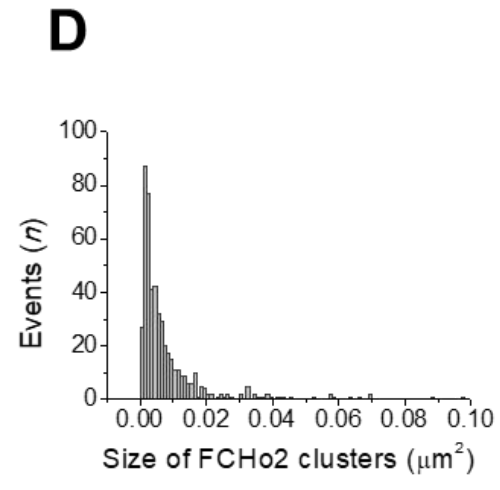

B
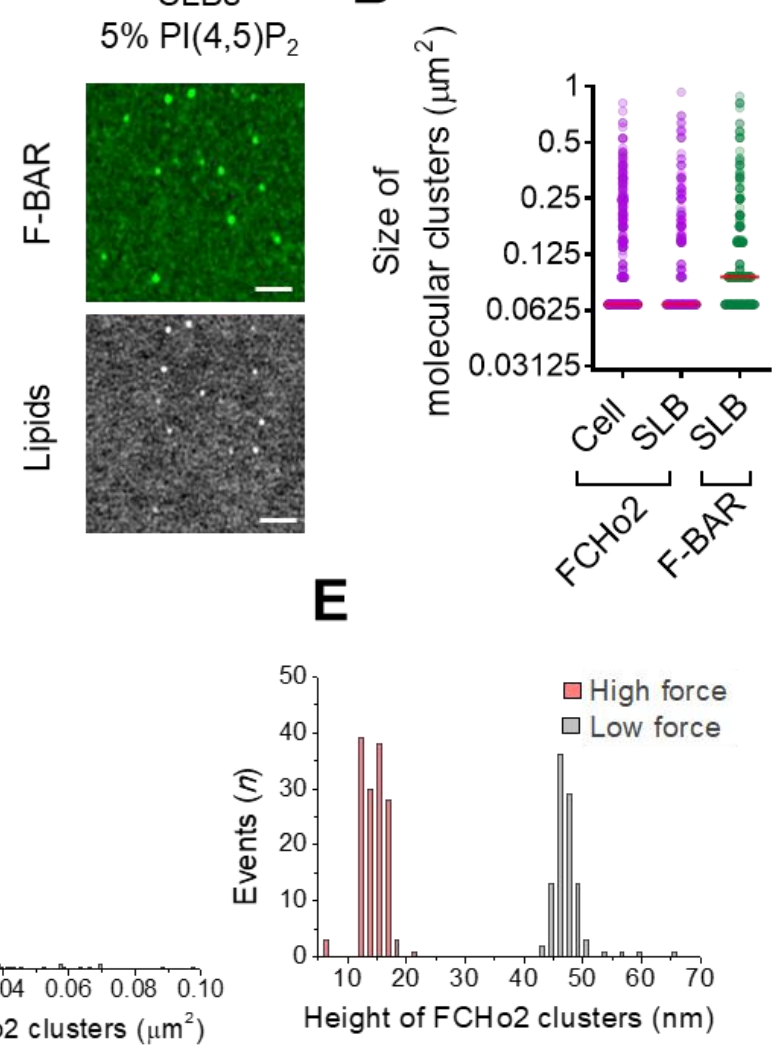

Figure 3. FCHo2 forms molecular clusters on PI(4,5) $\mathbf{P}_{2}$-containing bilayers. (A) Representative airyscan images of plasma membrane sheets or lipid bilayers doped with either $5 \%$ mol PI(4,5) $\mathrm{P}_{2}$ or $20 \% \mathrm{PS}$ (total negative charge is $20 \%$ ) and incubated with FCHo2-Alexa647 (magenta) or F-BAR-Alexa647 (green). Sale bar, $2 \mu \mathrm{m}$. (B) Size distribution of FCHo2 (magenta) and F-BAR (green) molecular clusters (in $\mu \mathrm{m}^{2}$ ) on plasma membrane sheets (cell) or lipid bilayers (SLB) containing $5 \%$ mol of $\mathrm{PI}(4,5) \mathrm{P}_{2}$. Median value is displayed in red. $\mathrm{n}=10793, \mathrm{n}=19287$, and $\mathrm{n}=7028$, as in the graph. (C) Representative AFM image of FCHo2 molecular clusters (white dashed region) on supported lipid bilayers containing 5\% mol PI(4,5) $\mathrm{P}_{2}$. (D) Size distribution of FCHo2 molecular clusters (in $\mu \mathrm{m}^{2}$ ) obtained from AFM images. (E) Height distribution of FCHo2 molecular clusters (in nm) at low (gray) and high (red) setpoint forces.

\section{FCHo2 forms ring-like shape assemblies on flat membranes}

To establish the biogenesis of FCHo2 clusters at the molecular level, we used high-speed AFM (HSAFM). Real-time imaging of the initial stages revealed the entire molecular process of FCHo2 cluster formation, from the binding of single $\mathrm{FCHo} 2$ homodimers to the growth of molecular clusters (Figure 4A). Representative time-lapse images and kymograph analysis along the dashed region at $\mathrm{t}=0 \mathrm{~s}$ showed that the binding of individual FCHo2 proteins engages an indentation of few $\mathrm{nm}$ in the lipid membrane adjacent to the protein surface (Figure 4B, green arrowheads), as delineated from the crosssection profile along the red dashed box in the corresponding kymograph (Figure 4A). The binding of FCHo2 rapidly prompted by the arrival of additional $\mathrm{FCHo} 2$ homodimers (as highlighted by white 
arrowheads in the kymograph). This stage of the process was characterized by minimal lateral interactions and the existence of contacts between adjacent FCHo2 homodimers, ultimately leading to a ring-like organization (Figure 4C). This dynamic reorganization that we named the "ring formation" step spanned over $\sim 80-100 \mathrm{~s}$.

The identification of individual proteins at the initial stages allowed us to extract the average dimension of the full-length protein interacting with flat membrane, which was $\sim 32 \pm 8 \mathrm{~nm}$ (Figure 4D) and in good agreement with the size of the F-BAR domain reported from electron microscopy micrographs (10). After the ring formation, we observed the growth of FCHo2 clusters through docking events that involved individual FCHo2 homodimers and the coalescence of adjacent FCHo2 rings (Figure 4A, white arrowheads). FCHo2 self-organization into hollow ring-like assemblies was particularly discernible at the growth front of FCHo2 molecular clusters (Figure 4E and magnified image). Although the entire formation of $\mathrm{FCHo} 2$ molecular clusters expanded over few tens of minutes, we found that the docking of individual proteins and rings to support the expansion of the cluster took place every $\sim 115 \pm 94 \mathrm{~s}$ (Figure 4F). Collectively, our results suggest that on flat membranes, FCHo2 exhibits an intrinsic ability to self-assemble into a ring-like shape molecular complex independently of the local protein density. 


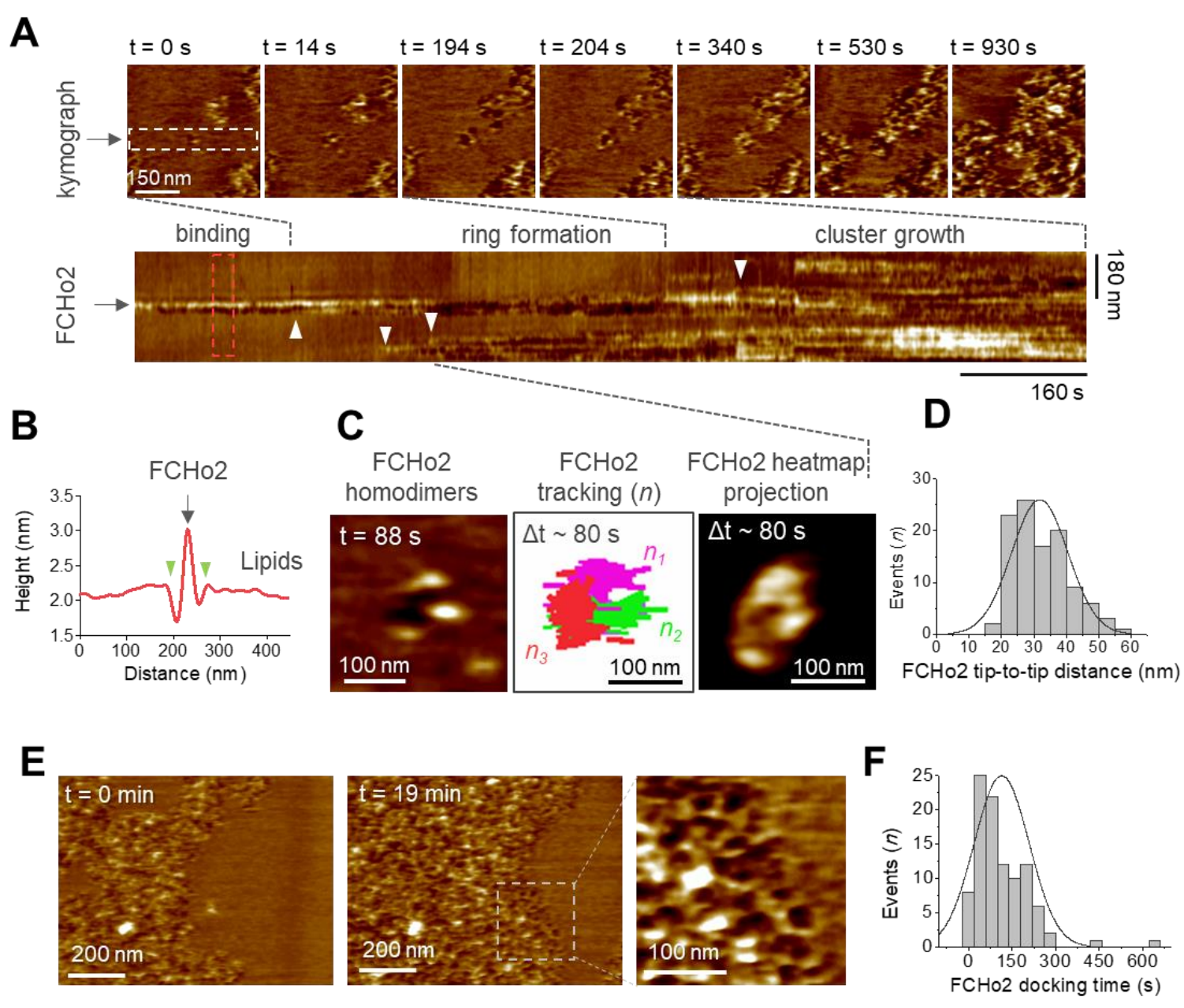

Figure 4. Molecular dynamics of FCHo2 self-assembly on PI(4,5)P2-containing membranes. (A) HS-AFM movie frames of the binding of $\mathrm{FCHo} 2$ on $5 \%$ mol PI(4,5) $\mathrm{P}_{2}$-containing membranes. Kymograph analysis performed on the white outline to display the representative stages of $\mathrm{FCHo} 2$ binding and self-assembly on flat membranes. White arrowheads highlight the docking of new FCHo2 homodimers to the growing molecular cluster. (B) Profile analysis along the red outline in A. Green arrowheads highlight the membrane invagination upon binding of FCHo2 on lipid membranes. (C) HS-AFM movie snapshot of the FCHo2 ring formation along with a representative tracking of individual homodimers $(n=3)$ and the heat map projection within a time interval, $\Delta \mathrm{t}$, of $\sim 80 \mathrm{~s}$. (D) Size distribution (in $\mathrm{nm}$ ) estimated from individual FCHo2 proteins before the cluster growth $(\mathrm{t} \leq 200 \mathrm{~s})$. (E) HS-AFM snapshots $(\mathrm{t}=0 \mathrm{~min}$ and $\mathrm{t}=19 \mathrm{~min})$ illustrating the growth of FCHo2 molecular clusters into ring-like shape protein patches. Magnified image corresponds to the dashed outline. (F) Distribution of the docking time (in s) of individual and ring-like FCHo2 assemblies during the cluster growth.

\section{PI(4,5) $P_{2}$ assists FCHo2 partitioning on curved membranes}

Because the transition from a flat surface to a dome-like invagination is a major step in the formation of clathrin-coated structures(2), we set out to monitor the organization of FCHo2 on curved membranes. To this end, we engineered arrays of $\mathrm{SiO}_{2}$ vertical nano-domes of radii $R \sim 150 \mathrm{~nm}$ using soft nanoimprint lithography (soft-NIL) (Figure 5A), as previously reported(25). We functionalized $\mathrm{SiO}_{2}$ nano- 
patterned substrates with supported lipid bilayers containing $20 \%$ of negatively charged lipids. First, we determined by airyscan microscopy the surface organization of the F-BAR domain and FCHo2 labeled with Alexa647 on nano-domes in the presence of lipid bilayers containing 5\% mol of PI(4,5) $\mathrm{P}_{2}$ (Figure $5 \mathrm{~B})$. The three-dimensional (3D) rendering of the protein signal relative to a reference marker to depict the nano-dome topography (DHPE lipid dye), showed that while the F-BAR domain is excluded from the nano-structure, the FCHo2 staining is well present at the base of the nano-dome (Figure 5B). We hypothesize that if the association of $\mathrm{FCHo} 2$ with $\mathrm{PI}(4,5) \mathrm{P}_{2}$ is essential for its localization on curved membranes, disrupting this interaction would change its spatial organization. Indeed, this was the case and replacing PI(4,5) $\mathrm{P}_{2}$ by PS (20\% mol PS) lead to complete distribution of FCHo2 all over the nanodome surface (Figure 5B, yellow).

To quantitatively estimate the preferential localization of FCHo2 on curved membranes, we computed for each nano-dome in patterned array the normalized maximal height of the protein signal relative to the DHPE lipid signal, which was used as a reference of the nano-dome height (see methods). Thus, a normalized maximal height close to $\sim 1.0$ indicates an increased probability of detecting the protein all over the nano-structure surface, whereas a value of $\sim 0$ would reflect an absence of preference for the nano-structure. On nano-domes functionalized with $\mathrm{PI}(4,5) \mathrm{P}_{2}$-containing membranes, we obtained a median value of the F-BAR domain maximal height of $\sim 0$ (Figure 5C, green), whereas FCHo2 displayed two preferential localizations but with a median height on nano-domes that was of $\sim 0.64$ (Figure 5C, magenta). Finally, on membranes containing PS we obtained that the maximal median height of FCHo2 on nano-domes was $\sim 1$ (Figure 5C, yellow), in agreement with the 3D renderings in Figure 5B.

Next, we determined whether FCHo2 partitioning on nano-domes might be affected by a heterogeneous distribution of $\mathrm{PI}(4,5) \mathrm{P}_{2}$ on curved membranes. To this end, we estimated the axial localization of the $\mathrm{PI}(4,5) \mathrm{P}_{2}$ dye relative to the DHPE lipid dye on nano-domes coated with 5\% $\mathrm{PI}(4,5) \mathrm{P}_{2}$-containing lipid bilayer (Figure 5D). The cross-section analysis showed an equivalent surface distribution of the $\mathrm{PI}(4,5) \mathrm{P}_{2}$ and DHPE lipid dye under our experimental conditions. To confirm that TF-TMR-PI $(4,5) \mathrm{P}_{2}$ signal was replicating the actual organization of the total pool of $\mathrm{PI}(4,5) \mathrm{P}_{2}$ on nano-domes, we analyzed the distribution of the $\mathrm{PH}$ domain of PLC $\delta 1 \mathrm{PH}(\mathrm{PLC} \delta 1)$, which is a well-established reporter of $\mathrm{PI}(4,5) \mathrm{P}_{2}(26)$. We obtained a median of the maximal height of the $\mathrm{PH}(\mathrm{PLC} \delta 1)$ of $\sim 1.0$, indicating a homogenous distribution along the nano-dome surface (Figure 5F). 
A

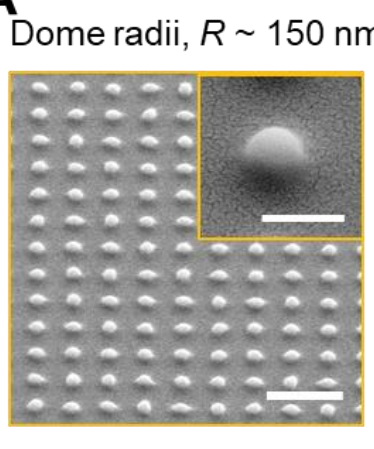

C

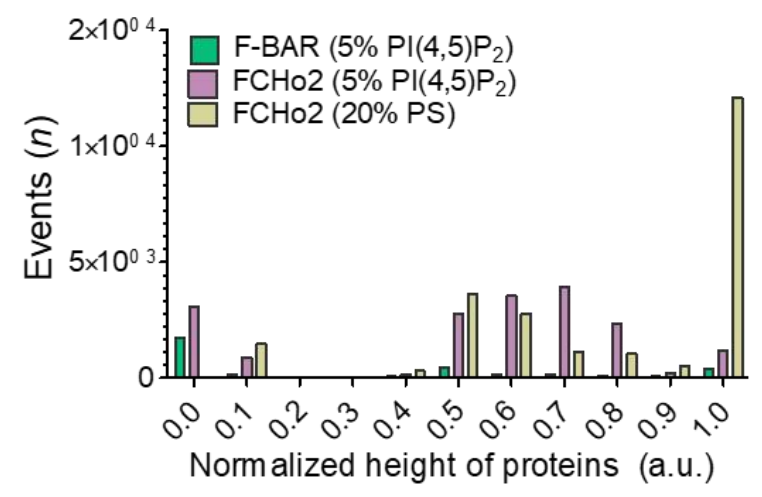

B
$5 \% \mathrm{Pl}(4,5) \mathrm{P}_{2}$
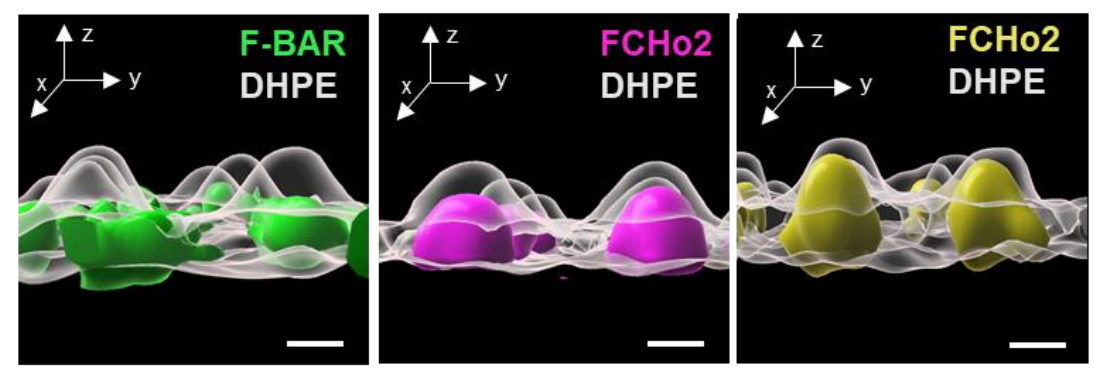

D

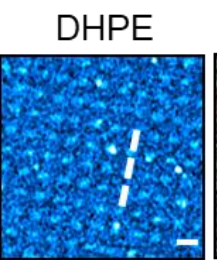

E
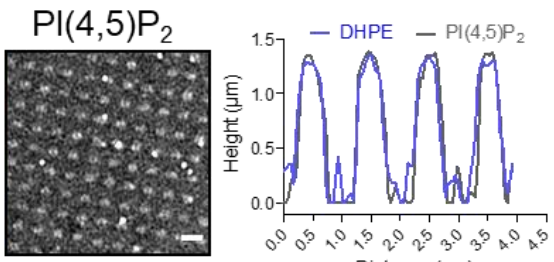

Distance $(\mu \mathrm{m})$

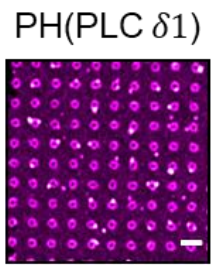

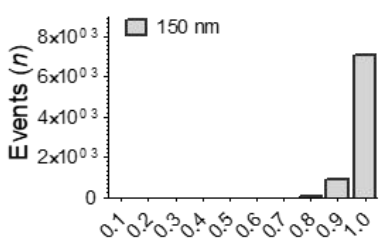

Normalized height of $\mathrm{PH}(\mathrm{PLC} 81)$ (a.u.)

Figure 5. PI(4,5) $\mathrm{P}_{2}$ assists the organization of $\mathrm{FCHo} 2$ on curved membranes. (A) $\mathrm{SEM}$ images of $\mathrm{SiO}_{2}$ substrates displaying an array of nano-domes with a radius, $R \sim 150 \mathrm{~nm}$ generated by soft-NIL. Scale bar, $2 \mu \mathrm{m}$. Inset, scale bar is $500 \mathrm{~nm}$. (B) Representative 3D renders of the surface organization of the F-BAR domain (green) and FCHo2 (magenta) on nano-domes functionalized with either $5 \%$ mol PI(4,5) $\mathrm{P}_{2}$ or $20 \%$ PS-containing membranes (yellow) relative to the DHPE lipid dye signal (gray). Scale bar, $400 \mathrm{~nm}$. (C) Distribution of the normalized maximal height of the surface distribution of the F-BAR domain (green) and FCHo2 (magenta) on nano-domes functionalized with 5\% mol of PI(4,5) $\mathrm{P}_{2}$ or $20 \%$ PS lipid bilayers (yellow). $\mathrm{n}=3300, \mathrm{n}=18145$ and $\mathrm{n}=23043$, respectively. (D) Left, $\mathrm{z}$-stack intensity projected airyscan images of the distribution of fluorescent DHPE and PI(4,5) $\mathrm{P}_{2}$ on nano-domes. Scale bar, $2 \mu \mathrm{m}$. Right, cross-section of the normalized height of DHPE (blue) and $\mathrm{PI}(4,5) \mathrm{P}_{2}$ (grey) lipid dyes obtained from the dashed line in the corresponding airyscan image. (E) Left, z-stack intensity projected airyscan images of the PH (PLC $\delta 1$ ) on nano-domes functionalized with $5 \%$ mol of PI(4,5) $\mathrm{P}_{2}$ lipid bilayers. Scale bar, $2 \mu \mathrm{m}$. Right, distribution of the normalized maximal height of the surface distribution of PH (PLC 81 ) on nano-domes. $\mathrm{n}=7974$.

\section{Discussion}

This study reports a molecular visualization of the docking and self-assembly of the endocytic protein FCHo2 on in vitro and cellular membranes (Figure 6). Our results show that $\mathrm{PI}(4,5) \mathrm{P}_{2}$ is a primary spatial regulator of the recruitment of $\mathrm{FCHo} 2$ by promoting its accumulation and sorting on flat and curved membranes. These observations support the model that multivalent interactions play an 
instrumental role in upholding the early stages of endocytosis. We show that FCHo2 oligomerization induces $\mathrm{PI}(4,5) \mathrm{P}_{2}$ clustering formation on cellular membranes that often co-localized with TfR and EGFR-positive puncta. This association was particularly remarkable in the case of the EGFR and agreed with the observation that electrostatic interaction of the polybasic motifs at the cytoplasmic tail of the EGFR mediates its clustering on PI(4,5)P2-enriched domains(16, 21, 27). FCHo1/2 is needed to recruit Eps15(7) and form FCHo1/2-Eps15 micrometer-scale domains on membranes(13). By driving PI(4,5) $\mathrm{P}_{2}$ clustering formation at the boundary of cargo receptors, $\mathrm{FCHo} 2$ is likely to improve the formation of a network of $\mathrm{PI}(4,5) \mathrm{P}_{2}$-interacting proteins. Indeed, our observations showing that $\mathrm{FCHo} 2$ intensifies the formation of clathrin-positive assemblies on in vitro membranes through $\mathrm{PI}(4,5) \mathrm{P}_{2}$-rich interfaces accords with previous studies pointing that clustering of Fcho1/2Eps15/AP2 primes endocytosis(8). We discerned clathrin-positive puncta in lower FCHo2 concentrations but not in PI(4,5)P2-depleted membranes, which agrees with the observation that AP2 can create its local pool of $\mathrm{PI}(4,5) \mathrm{P}_{2}(28)$, although it requires $\mathrm{PI}(4,5) \mathrm{P}_{2}$ for its localization and activation at the plasma membrane $(29,30)$. Our measurements point that local $\mathrm{PI}(4,5) \mathrm{P}_{2}$ enrichment induced by $\mathrm{FCHo} 2$ might operate as a complementary mechanism to enhance $\mathrm{PI}(4,5) \mathrm{P}_{2}$-mediated interactions at endocytic sites.

This work provides the first evidence that FCHo2 self-assembles into ring-like molecular complexes on flat membranes. HS-AFM movies show that the ring formation process is relatively fast and takes place within less than $100 \mathrm{~s}$, which might be compatible with the temporal scales reported during clathrin-coat assembly(1). This type of ring organization agrees with a side-lying conformation proposed for F-BAR scaffolds at low protein densities on flat surfaces(10) and the partitioning of FCHo1/2 at the edges of flat clathrin lattices(31). Lateral contacts stabilize the self-assembly of F-BAR domains on membrane tubules $(10,32)$. Our investigations point out that lateral interactions might occur on flat surfaces. We observed the anisotropic growth and formation of FCHo2 molecular clusters in the absence of other endocytic proteins. The formation of FCHo2 molecular complexes might confer structural stability to the ring-like organization and, importantly, show that F-BAR proteins can moderately bend flat membranes at high protein densities. 


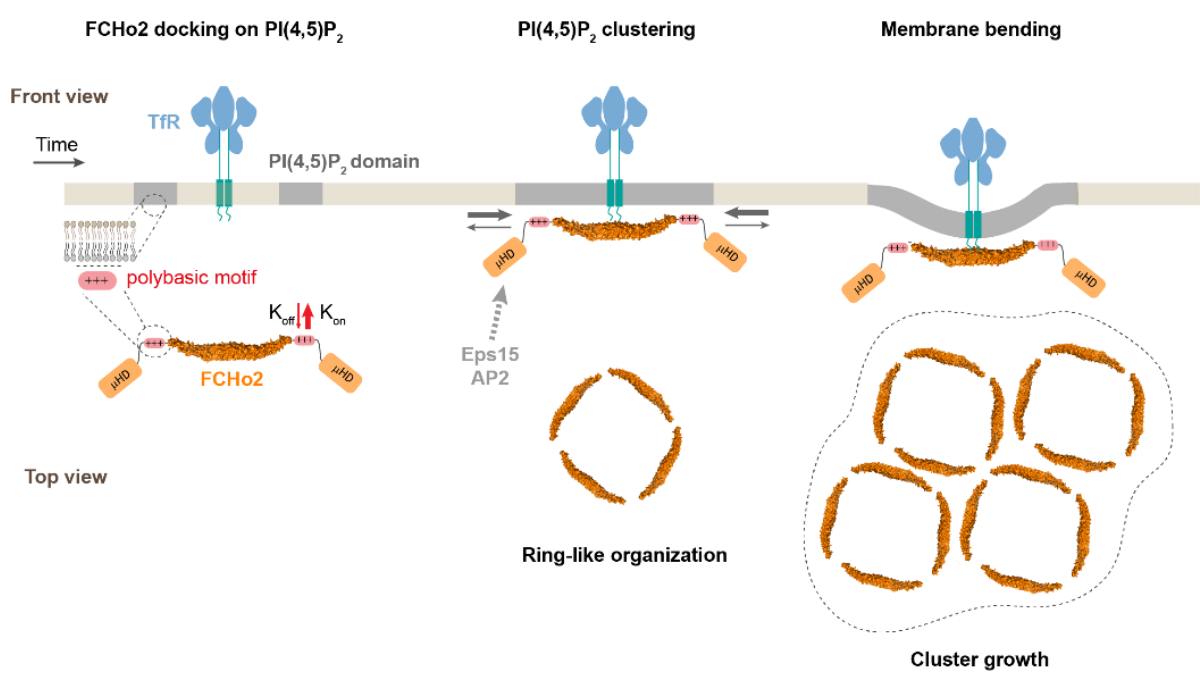

Figure 6. Model of FCHo2 docking and self-assembly on membranes. FCHo2 recruitment of membranes is mediated by $\mathrm{PI}(4,5) \mathrm{P}_{2}$. Increase in the local $\mathrm{PI}(4,5) \mathrm{P}_{2}$ concentration enhances the spatial accumulation of $\mathrm{FCHo} 2$ through multivalent interaction between the positively charged surface of the F-BAR domain and polybasic motif and $\mathrm{PI}(4,5) \mathrm{P}_{2}$ molecules. Binding of FCHo2 on cellular membranes is convoyed by $\mathrm{PI}(4,5) \mathrm{P}_{2}$ clustering formation at the boundary of clathrin-regulated cargo receptors and the self-assembly of $\mathrm{FCHo} 2$ into a ring-like shape protein complex. As a result of this singular organization, the local accumulation of $\mathrm{PI}(4,5) \mathrm{P}_{2}$ and $\mu \mathrm{HD}$ of $\mathrm{FCHo} 2$ is likely to facilitate the formation of an interacting network with downstream partners, such as Eps15 and AP2 (7, 8), ultimately leading to the assembly of clathrin structures on a $\mathrm{PI}(4,5) \mathrm{P}_{2-}$ rich interface that could be amplified by phosphoinositides metabolizing enzymes(3). In the absence of endocytic partners, FCHo2 rings grow into sub-micrometric molecular clusters leading to membrane bending and the partitioning on curved membranes.

\section{Conclusions}

In conclusion, our study provides a molecular picture of the recruitment and self-assembly of the early endocytic protein $\mathrm{FCHo} 2$ on membranes. While $\mathrm{PI}(4,5) \mathrm{P}_{2}$-enriched domains act as docking sites to recruit FCHo2 on in vitro and cellular membrane, we found that the binding of FCHo2 promotes the local accumulation of $\mathrm{PI}(4,5) \mathrm{P}_{2}$ at the vicinity of clathrin-regulated cargo receptors. As a result, in the absence of phosphoinositides-metabolizing enzymes, FCHo2 can enhance the formation of clathrin structures through a local $\mathrm{PI}(4,5) \mathrm{P}_{2}$ enrichment, which could explain previous studies showing that FCHo1/2 depletion slows down the progression of cargo-loaded clathrin structures $(8,9,12,33)$. Because FCHo2 is among the early proteins recruited at endocytic sites, the discovery that FCHo2 selfassembles into rings convoyed by membrane bending and $\mathrm{PI}(4,5) \mathrm{P}_{2}$ accumulation provides a fundamental understanding of the initiating mechanism of clathrin-mediated endocytosis(2). 


\section{Materials and Methods}

\section{Lipids and reagents}

Natural and synthetic phospholipids, including POPC, POPS, Egg-PC, Brain-PS, Brain-PI(4,5) $\mathrm{P}_{2}$ and fluorescent TopFluor-TMR-PtdIns(4,5) $\mathrm{P}_{2}$ are from Avanti Polar Lipids, Inc. Oregon green 488-DHPE and Alexa Fluor 647 Maleimide labelling kit are from Invitrogen. Atto647N-DOPE and polyclonal rabbit anti-clathrin heavy chain (dilution 1:100; Cat. Nr. HPA059143) was from Sigma. Monoclonal rabbit anti-EGFR (dilution 1:1000; Cat. Nr. 4267) and polyclonal rabbit anti-phospho-EGFR (Tyr1045) (dilution 1:1000; Cat. Nr. 2237) were from Cell Signaling.

\section{EGFR-GFP and TfR-GFP stable cell lines}

pRRL.sin.cPPT.SFFV-EGFP/IRES-puro was kindly provided by C. Goujon (IRIM, CNRS UMR9004, Montpellier, France), the EGFR-GFP vector was a gift from Alexander Sorkin, (Addgene plasmid \#32751) and the pBa.TfR.GFP vector was a gift from Gary Banker \& Marvin Bentley (Addgene plasmid \# 4506). The GFP was replaced by a GFP-delta-ATG using these primers: 5'gtatatatatGGATCCGTGAGCAAGGGCGAGGAG-3' and 5'-CTCACATTGCCAAAAGACG-3'. GFP-delta-ATG fragment replaced the GFP- fragment in pRRL.sin.cPPT.SFFV-EGFP/IRES-puro using a BamHI-XhoI digestion. EGFR was amplified with these primers: 5'caaatatttgcggccgcATGCGACCCTCCGGGACG-3' ${ }^{\prime}$ and gtataccggttgaacctccgccTGCTCCAATAAATTCACTGCTTTGTGG-3' and cloned into pRRL.sin.cPPT.SFFV-EGFP-delta-ATG/IRES-puro using NotI-AgeI to generate a fused EGFR-GFP protein.

Lentiviral vector stocks were obtained by polyethylenimine (PEI) -mediated multiple transfection of $293 \mathrm{~T}$ cells in 6-well plates with vectors expressing Gag-Pol (8.91), the mini-viral genome (pRRL.sin.cPPT.SFFV-EGFR-GFP/IRES-puro) and the Env glycoprotein of VSV (pMD.G) at a ratio of 1:1:0.5. The culture medium was changed $6 \mathrm{hr}$ post-transfection and lentivectors containing supernatants harvested $48 \mathrm{hr}$ later, filtered and stored at $-80^{\circ} \mathrm{C}$.

The mini-viral genome (pRRL.sin.cPPT.SFFV-TFR-GFP/IRES-puro) and the corresponding lentivectors were generated as detailed in the case of the EGFR-GFP.

To generate a stable cell line HT1080 expressing EGFR-GFP or TfR-GFP, HT1080 cells (a gift from N. Arhel, IRIM, CNRS UMR9004, Montpellier, France) were transduced in 6-well plates using the supernatant of one 6-well plates of the lentiviral stock production detailed above. The culture medium was changed $6 \mathrm{hr}$ post-transduction. Puromycin $(1 \mu \mathrm{g} / \mathrm{ml})$ was added $48 \mathrm{~h}$ after transduction. The 
percentage of GFP-expressing cells was enumerated by flow cytometry $72 \mathrm{hr}$ after selection under puromycin.

HT1080 cells constitutively expressing the EGFR-GFP or TfR-GFP were cultured in DMEM GlutaMAX supplemented with $10 \%$ fetal calf serum, $100 \mathrm{U} \cdot \mathrm{mL}^{-1}$ of penicillin and streptomycin and $1 \mu \mathrm{g} \cdot \mathrm{ml}^{-1}$ of puromycin at $37^{\circ} \mathrm{C}$ in $5 \% \mathrm{CO}_{2}$. Cell lines were tested negative for mycoplasma.

\section{Protein purification and protein labeling}

pGEX-6P-1 vector coding for the mouse full-length FCHo2 (aa 1-809) and F-BAR domain (aa 1-262) and human FBP17 (aa 1-592) were obtained from H.T McMahon (MRC Laboratory of Molecular Biology, Cambridge, UK). Proteins were subcloned into a pET28a vector with a PreScission protease cleaving site. Proteins were expressed in Rosetta 2 bacteria and purified by affinity chromatography using a HiTrap ${ }^{\mathrm{TM}}$ chelating column (GE Healthcare) according to the manufacturer's instructions in 50 $\mathrm{mM}$ Tris at $\mathrm{pH} 8.0,100 \mathrm{mM} \mathrm{NaCl}$. Proteins were expressed overnight at $18^{\circ} \mathrm{C}$ using $1 \mathrm{mM}$ IPTG. Proteins were then dialyzed overnight in a Slide-A-Lyzer dialysis cassette (MWCO 10,000) before Alexa Fluor 647 maleimide labelling following the protocol described by the manufacturer (Invitrogen). Protein concentrations were measured using a Bradford assay (Biorad).

Recombinant GST-eGFP-PH-domain (PLC 81$)$ detecting PI(4,5) $\mathrm{P}_{2}$ was purified as described in(25).

\section{Xenopus laevis egg extracts}

Laid eggs are rinsed twice in XB Buffer $(100 \mathrm{mM} \mathrm{KCl}, 1 \mathrm{mM} \mathrm{MgCl} 2,0.1 \mathrm{mM} \mathrm{CaCl} 2,50 \mathrm{mM}$ sucrose and $10 \mathrm{mM}$ HEPES at $\mathrm{pH} 7.7$ ) and subsequently dejellied with $2 \%$ cysteine solution $\mathrm{pH} 7.8$. Once dejellied they are extensively rinsed with XB buffer to completely eliminate cysteine solution.

Eggs are then recovered from a Petri dish and treated with Ca2+ Ionophore (final concentration $2 \mu \mathrm{g} / \mathrm{ml}$ ) and 35 minutes later, they are crushed by centrifugation for $20 \mathrm{~min}$ at $10,000 \mathrm{~g}$ at $4{ }^{\circ} \mathrm{C}$. The cytoplasmic layer is collected and supplemented with Cytochalasin B (50ug/ml) Aprotinin (5ug/ml), Leupeptin $(5 \mathrm{ug} / \mathrm{ml})$ and $10 \mathrm{mM}$ Creatin Phosphate. Cytoplasmic extract is centrifuged again for $20 \mathrm{~min}$ at 10,000g. Extract are frozen and then used as described in Figure 2.

The energy mix consisted of $1.5 \mathrm{mM}$ ATP, $0.15 \mathrm{mM} \mathrm{GTP} \gamma \mathrm{S}, 16.7 \mathrm{mM}$ creatine phosphate and creatine phosphokinase $16.7 \mathrm{U} \cdot \mathrm{ml}^{-1}$, as previously reported(34) 


\section{Supported lipid bilayers}

Lipid mixtures consisted of: $80-85 \%$ Egg-PC, $10-15 \%$ Brain-PS and 5-10\% of Brain-PI(4,5) $\mathrm{P}_{2}$. The amount of total negatively charged lipids was kept to $20 \%$ for any of the mixtures containing phosphoinositides at the expenses of Brain-PS. If needed, fluorescent lipids were added to $0.2 \%$.

For fluorescence microscopy experiments, supported lipid bilayers were prepared as described in(35). Experiments were performed by injecting $15 \mu \mathrm{L}$ of buffer (20 mM Tris, $\mathrm{pH} 7.4,150 \mathrm{mM} \mathrm{NaCl}$ and 0.5 $\mathrm{mg} \cdot \mathrm{ml}^{-1}$ of casein). Supported lipid bilayers were imaged on a Zeiss LSM880 confocal microscope.

For HS-AFM experiments, supported lipid bilayers were prepared following the method described in(36). Briefly, large unilamellar vesicles (LUVs, diameter $\sim 100 \mathrm{~nm}$ ) were obtained by extrusion of multilamellar vesicles of 85\% POPC, 10\% POPS and 5\% Brain-PI(4,5) $\mathrm{P}_{2}$ in $20 \mathrm{mM}$ Hepes, pH 7.4, 150 $\mathrm{mM} \mathrm{NaCl}$. LUVs were supplemented with $20 \mathrm{mM}$ of $\mathrm{CaCl}_{2}$ and deposited onto freshly cleaved mica disks. Samples were incubated for $20 \mathrm{~min}$ at $60^{\circ} \mathrm{C}$ and extensively rinsed with $20 \mathrm{mM}$ Hepes, pH 7.4, $150 \mathrm{mM} \mathrm{NaCl}, 20 \mathrm{mM}$ EDTA. Finally, bilayers were rinsed and keep under the imaging buffer, $20 \mathrm{mM}$ Hepes, pH 7.4, $150 \mathrm{mM} \mathrm{NaCl}$.

\section{Plasma membrane sheets}

Unroofing of HT1080 cells stably expressing the EGFR-GFP was performed by tip sonication as reported in(37). Cells were rinsed three times in cold Ringer buffer supplemented with $\mathrm{Ca}^{2+}(155 \mathrm{mM}$ $\mathrm{NaCl}, 3 \mathrm{mM} \mathrm{KCl}, 3 \mathrm{mM} \mathrm{NaH} 2 \mathrm{PO}_{4}, 5 \mathrm{mM}$ HEPES, 10mM glucose, $2 \mathrm{mM} \mathrm{CaCl}_{2}, 1 \mathrm{mM} \mathrm{MgCl}_{2}, \mathrm{pH} 7.2$ ), then immersed $10 \mathrm{~s}$ in $\mathrm{Ca}^{2+}$-free Ringer buffer containing $0.5 \mathrm{mg} \cdot \mathrm{mL}^{-1}$ poly-L-lysine. Cells were unroofed by scanning the coverslip with the tip sonicator at $10 \%$ of power under $\mathrm{HKMgE}$ buffer consisting of $70 \mathrm{mM} \mathrm{KCl}, 30 \mathrm{mM}$ HEPES, $5 \mathrm{mM} \mathrm{MgCl} 2,3 \mathrm{mM}$ EGTA, $\mathrm{pH}$ 7.2. Unroofed cells were kept in $\mathrm{HKMgE}$ buffer. Fluorescent labeling of plasma membrane sheets was performed immediately after unroofing by incubating the sample with $100 \mathrm{nmol}$ of TopFluor-TMR-PtdIns $(4,5) \mathrm{P}_{2}$ suspended in $0.2 \%$ of absolute ethanol during $5 \mathrm{~min}$, as reported in(38). Then, samples were extensively rinsed with HKMgE buffer and immediately imaged under the Zeiss LSM880 confocal microscope. Before addition of $1 \mu \mathrm{M}$ of FCHo2-Alexa647, unroofed cells were rinsed with $\mathrm{HKMgE}$ buffer supplemented with 0.5 $\mathrm{mg} \cdot \mathrm{ml}^{-1}$ of casein.

\section{Immunofluorescence}

Supported lipid bilayers were fixed in 3.7\% PFA in PBS for $2 \mathrm{~min}$ at room temperature, then rinsed in PBS twice. Samples were stained for the primary antibody for $45 \mathrm{~min}$ at room temperature in $1 \%$ BSA. Then, the secondary antibody was incubated for 45 min. Finally, samples were extensively rinsed in 
PBS, then in sterile water and mounted with a Mowiol ${ }^{\circledR}$ 4-88 mounting medium (Polysciences, Inc.). Montage was allowed to solidify in the dark for $48 \mathrm{~h}$ before microscope acquisitions.

\section{Silica thin film nanostructuration}

$\mathrm{SiO}_{2}$ vertical nanostructures were prepared on conventional borosilicate coverslips with precision thickness No. $1.5(0.170 \pm 0.005 \mathrm{~mm})$, as previously reported $(25,39)$. Briefly, Si masters were elaborated using LIL Lithography as detailed in $(39,40)$. PDMS (polydimethylsiloxane) reactants (90 w\% RTV141A; 10 w\% RTV141B from BLUESIL) were transferred onto the master and dried at $70{ }^{\circ} \mathrm{C}$ for $1 \mathrm{~h}$ before unmolding.

Silica precursor solution was prepared by adding $4.22 \mathrm{~g}$ tetraethyl orthosilicate (TEOS) into $23.26 \mathrm{~g}$ absolute ethanol, then $1.5 \mathrm{~g} \mathrm{HCl}$ (37\%), and stirring the solution for $18 \mathrm{~h}$. The final molar composition was TEOS:HCl:EtOH=1:0.7:25. All the chemicals were from Sigma. Gel films were obtained by dipcoating the coverslips with a ND-DC300 dip-coater (Nadetech Innovations) equipped with an EBC10 Miniclima device to control the surrounding temperature and relative humidity to $20^{\circ} \mathrm{C}$ and $45-50 \%$, respectively. The thickness of film was controlled by the withdrawal rate at $300 \mathrm{~mm} / \mathrm{min}$. After dipcoating, gel films were consolidated at $430^{\circ} \mathrm{C}$ for $5 \mathrm{~min}$. Then, a new layer of the same solution was deposited under the same conditions for printing with the PDMS mold. After imprinting, the samples were transferred to a $70{ }^{\circ} \mathrm{C}$ oven for $1 \mathrm{~min}$ and then to a $140{ }^{\circ} \mathrm{C}$ for $2 \mathrm{~min}$ to consolidate the xerogel films before peeling off the PDMS mold. Finally, the sol-gel replicas were annealed at $430{ }^{\circ} \mathrm{C}$ for 10 min for consolidation.

\section{Fluorescence microscopy}

Images were acquired on a Zeiss LSM880 Airyscan confocal microscope (MRI facility, Montpellier). Excitation sources used were: $405 \mathrm{~nm}$ diode laser, an Argon laser for $488 \mathrm{~nm}$ and $514 \mathrm{~nm}$ and a Helium/Neon laser for $633 \mathrm{~nm}$. Acquisitions were performed on a 63x/1.4 objective. Multidimensional acquisitions were acquired via an Airyscan detector (32-channel GaAsP photomultiplier tube (PMT) array detector). 3D images were acquired by fixing a $0.15 \mu \mathrm{m}$ z-step in order to cover the entire surface of vertical nanostructures.

\section{HS-AFM imaging}

HS-AFM movies were acquired with an HS-AFM (SS-NEX, Research Institute of Biomolecule Metrology, Tsukuba, Japan) equipped with a superluminescent diode (wavelength, $750 \mathrm{~nm}$; EXS 7505B001, Exalos, Schlieren, Switzerland) and a digital high-speed lock-in Amplifier (Hinstra, Transcommers, Budapest, Hungary)(41) following the protocol detailed at(42). Scanning was performed using USC-1.2 cantilevers featuring an electron beam deposition tip (NanoWorld, Neuchâtel, 
Switzerland) with a nominal spring constant $\mathrm{k}=0.15 \mathrm{~N} / \mathrm{m}$, resonance frequency $\mathrm{f}(\mathrm{r})=600 \mathrm{kHz}$, and quality factor $\mathrm{Qc} \approx 2$ under liquid conditions. For high-resolution imaging, the electron beam deposition tip was sharpened by helium plasma etching using a plasma cleaner (Diener Electronic, Ebhausen, Germany). Images were acquired in amplitude modulation mode at the minimal possible applied force that enables good quality of imaging under optical feedback parameters.

\section{Image processing and quantification}

Line profiles of the fluorescence intensities were done using ImageJ(43) and the kymographs were made using the Kymograph plugin (http://www.embl.de/eamnet/html/body_kymograph.html).

Protein binding was quantified by measuring the mean grey value of the protein channel that was then normalized by the mean gray value of the membrane intensity (as indicated by the TF-TMR-PI $(4,5) \mathrm{P}_{2}$ fluorescence) in the same image. Mean gray values were measured once protein binding reached the steady-state, which was estimated from the binding kinetics to be $<4$ min. Protein binding was averaged from 3 experimental replicates. Mean gray values were measured using ImageJ. Concentrations and confocal parameters were kept constant between experiments and samples.

The automatic analysis of images to determine molecular clusters was performed with ImageJ(43). Spots of different sizes are detected using a scale space spot detection (44) and overlapping spots are merged. The LoG filter of the FeatureJ plugin(45) is used to create the scale space. Starting points are detected as local minima of the minimum projection and as minima on the smallest scale. A simplified linking scheme is applied that looks for minima along the scales for each starting point within the radius of the spot on the given scale. Two spots are merged if at least 20 percent of the surface of one spot is covered by the other. The noise tolerance for the spot detection is determined manually for each series of input images.

To determine the height of each protein surface on $\mathrm{SiO}_{2}$ vertical nanostructures, we applied an automated image analysis procedure that calculates the statistics about the average maximal height of the signal in each channel of interest, as previously described(25). The analysis has been implemented as an ImageJ(43) macro toolset(46).

HS-AFM images were processed using Gwyddion, an open-source software for SPM data analysis, and WSxM(47).

\section{Data representation and statistical analysis}

Representation of cross-section analysis and recruitment curves was performed using Origin software. 
3D rendering of Airyscan images was generated with the 3/4D visualization and analysis software Imaris (Oxford Instruments).

Statistical analysis was performed using Prism GraphPad software.

\section{References}

1. M. J. Taylor, D. Perrais, C. J. Merrifield, A High Precision Survey of the Molecular Dynamics of Mammalian Clathrin-Mediated Endocytosis. PLoS Biol 9, e1000604 (2011).

2. V. Haucke, M. M. Kozlov, Membrane remodeling in clathrin-mediated endocytosis. J Cell Sci 131, jcs216812 (2018).

3. Y. Posor, M. Eichhorn-Grünig, V. Haucke, Phosphoinositides in endocytosis. Biochimica et Biophysica Acta (BBA) - Molecular and Cell Biology of Lipids 1851, 794-804 (2015).

4. G. J. Doherty, H. T. McMahon, Mechanisms of Endocytosis. Annu. Rev. Biochem. 78, 857-902 (2009).

5. M. P. Sheetz, Cell control by membrane-cytoskeleton adhesion. Nat Rev Mol Cell Biol 2, 392396 (2001).

6. C. Godlee, M. Kaksonen, From uncertain beginnings: Initiation mechanisms of clathrin-mediated endocytosis. J Cell Biol 203, 717-725 (2013).

7. W. M. Henne, et al., FCHo Proteins Are Nucleators of Clathrin-Mediated Endocytosis. Science 328, 1281-1284 (2010).

8. L. Ma, et al., Transient Fcho1/2-Eps15/R-AP-2 Nanoclusters Prime the AP-2 Clathrin Adaptor for Cargo Binding. Developmental Cell 37, 428-443 (2016).

9. G. Hollopeter, et al., The membrane-associated proteins FCHo and SGIP are allosteric activators of the AP2 clathrin adaptor complex. eLife 3, e03648 (2014).

10. A. Frost, et al., Structural Basis of Membrane Invagination by F-BAR Domains. Cell 132, 807817 (2008).

11. W. M. Henne, et al., Structure and Analysis of FCHo2 F-BAR Domain: A Dimerizing and Membrane Recruitment Module that Effects Membrane Curvature. Structure 15, 839-852 (2007).

12. P. K. Umasankar, et al., Distinct and separable activities of the endocytic clathrin-coat components Fcho1/2 and AP-2 in developmental patterning. Nat Cell Biol 14, 488-501 (2012).

13. K. J. Day, et al., Liquid-like protein interactions catalyse assembly of endocytic vesicles. Nat Cell Biol 23, 366-376 (2021).

14. J. P. Zewe, et al., Probing the subcellular distribution of phosphatidylinositol reveals a surprising lack at the plasma membrane. Journal of Cell Biology 219 (2020). 
15. T. Itoh, et al., Dynamin and the Actin Cytoskeleton Cooperatively Regulate Plasma Membrane Invagination by BAR and F-BAR Proteins. Developmental Cell 9, 791-804 (2005).

16. H. Kolds $\varnothing$, D. Shorthouse, J. Hélie, M. S. P. Sansom, Lipid Clustering Correlates with Membrane Curvature as Revealed by Molecular Simulations of Complex Lipid Bilayers. PLoS Comput Biol 10, e1003911 (2014).

17. A. Honigmann, et al., Phosphatidylinositol 4,5-bisphosphate clusters act as molecular beacons for vesicle recruitment. Nat Struct Mol Biol 20, 679-686 (2013).

18. G. van den Bogaart, et al., Membrane protein sequestering by ionic protein-lipid interactions. Nature 479, 552-555 (2011).

19. H. Zhao, et al., Membrane-Sculpting BAR Domains Generate Stable Lipid Microdomains. Cell Reports 4, 1213-1223 (2013).

20. L. Picas, et al., BIN1/M-Amphiphysin2 induces clustering of phosphoinositides to recruit its downstream partner dynamin. Nat Commun 5, 5647 (2014).

21. Y. Wang, et al., Regulation of EGFR nanocluster formation by ionic protein-lipid interaction. Cell Res 24, 959-976 (2014).

22. L. M. Traub, J. S. Bonifacino, Cargo Recognition in Clathrin-Mediated Endocytosis. Cold Spring Harbor Perspectives in Biology 5, a016790-a016790 (2013).

23. A. Walrant, D. S. Saxton, G. P. Correia, J. L. Gallop, "Triggering actin polymerization in Xenopus egg extracts from phosphoinositide-containing lipid bilayers" in Methods in Cell Biology, (Elsevier, 2015), pp. 125-147.

24. F. Daste, et al., Control of actin polymerization via the coincidence of phosphoinositides and high membrane curvature. J. Cell Biol. 216, 3745-3765 (2017).

25. T. Sansen, et al., Mapping Cell Membrane Organization and Dynamics Using Soft Nanoimprint Lithography. ACS Appl. Mater. Interfaces, acsami.0c05432 (2020).

26. M. A. Lemmon, K. M. Ferguson, R. O’Brien, P. B. Sigler, J. Schlessinger, Specific and highaffinity binding of inositol phosphates to an isolated pleckstrin homology domain. Proceedings of the National Academy of Sciences 92, 10472-10476 (1995).

27. K. B. Abd Halim, H. Koldsø, M. S. P. Sansom, Interactions of the EGFR juxtamembrane domain with PIP2-containing lipid bilayers: Insights from multiscale molecular dynamics simulations. Biochimica et Biophysica Acta (BBA) - General Subjects 1850, 1017-1025 (2015).

28. M. Krauss, V. Kukhtina, A. Pechstein, V. Haucke, Stimulation of phosphatidylinositol kinase type I-mediated phosphatidylinositol (4,5)-bisphosphate synthesis by AP-2 -cargo complexes. Proceedings of the National Academy of Sciences 103, 11934-11939 (2006).

29. Z. Kadlecova, et al., Regulation of clathrin-mediated endocytosis by hierarchical allosteric activation of AP2. Journal of Cell Biology 216, 167-179 (2017).

30. S. Höning, et al., Phosphatidylinositol-(4,5)-Bisphosphate Regulates Sorting Signal Recognition by the Clathrin-Associated Adaptor Complex AP2. Molecular Cell 18, 519-531 (2005).

31. K. A. Sochacki, A. M. Dickey, M.-P. Strub, J. W. Taraska, Endocytic proteins are partitioned at the edge of the clathrin lattice in mammalian cells. Nat Cell Biol 19, 352-361 (2017). 
32. C. Mim, et al., Structural Basis of Membrane Bending by the N-BAR Protein Endophilin. Cell 149, 137-145 (2012).

33. E. E. Mulkearns, J. A. Cooper, FCH domain only-2 organizes clathrin-coated structures and interacts with Disabled-2 for low-density lipoprotein receptor endocytosis. $M B o C$ 23, 1330-1342 (2012).

34. M. Wu, et al., Coupling between clathrin-dependent endocytic budding and F-BAR-dependent tubulation in a cell-free system. Nat Cell Biol 12, 902-908 (2010).

35. J. A. Braunger, C. Kramer, D. Morick, C. Steinem, Solid Supported Membranes Doped with PIP 2 : Influence of Ionic Strength and $\mathrm{pH}$ on Bilayer Formation and Membrane Organization. Langmuir 29, 14204-14213 (2013).

36. L. Picas, et al., Preferential insertion of lactose permease in phospholipid domains: AFM observations. Biochimica et Biophysica Acta (BBA) - Biomembranes 1798, 1014-1019 (2010).

37. J. Heuser, The Production of 'Cell Cortices' for Light and Electron Microscopy. Traffic 1, 545552 (2000).

38. V. Mueller, et al., STED Nanoscopy Reveals Molecular Details of Cholesterol- and Cytoskeleton-Modulated Lipid Interactions in Living Cells. Biophysical Journal 101, 1651-1660 (2011).

39. Q. Zhang, et al., Micro/Nanostructure Engineering of Epitaxial Piezoelectric $\alpha$-Quartz Thin Films on Silicon. ACS Appl. Mater. Interfaces 12, 4732-4740 (2020).

40. Q. Zhang, et al., Tailoring the crystal growth of quartz on silicon for patterning epitaxial piezoelectric films. Nanoscale Adv., 10.1039.C9NA00388F (2019).

41. A. Colom, I. Casuso, F. Rico, S. Scheuring, A hybrid high-speed atomic force-optical microscope for visualizing single membrane proteins on eukaryotic cells. Nat Commun 4, 2155 (2013).

42. F. Zuttion, L. Redondo-Morata, A. Marchesi, I. Casuso, "High-Resolution and High-Speed Atomic Force Microscope Imaging" in Nanoscale Imaging: Methods and Protocols, Methods in Molecular Biology., Y. L. Lyubchenko, Ed. (Springer, 2018), pp. 181-200.

43. C. A. Schneider, W. S. Rasband, K. W. Eliceiri, NIH Image to ImageJ: 25 years of image analysis. Nat Methods 9, 671-675 (2012).

44. T. Lindeberg, Scale-Space Theory in Computer Vision (Springer US, 1994) https:/doi.org/10.1007/978-1-4757-6465-9 (October 14, 2020).

45. FeatureJ (October 14, 2020).

46.

https://github.com/MontpellierRessourcesImagerie/imagej_macros_and_scripts/wiki/MRI_Hei ghts_of_Surfaces_Tools.

47. I. Horcas, et al., WSXM: A software for scanning probe microscopy and a tool for nanotechnology. Review of Scientific Instruments 78, 013705 (2007). 


\section{Acknowledgements}

The authors thank H.T. McMahon for kindly providing F-BAR domain protein constructs. C. Goujon and O. Moncorgé for helping in the transduction of HT1080 cells. C. Holuka for assistance with plasma membrane sheets. P. Maiuri for assistance in data analysis. J.B. Manneville for critical reading of the manuscript and discussion. S. Roche, C. Favard, and D. Muriaux for scientific discussions. We acknowledge the imaging facility MRI, member of the national infrastructure France-BioImaging infrastructure supported by the French National Research Agency (ANR-10-INBS-04, «Investments for the future»). L.P. acknowledges the ATIP-Avenir program (AO-2016) and ANR-18-CE13-0015-02 for financial support. A.C-G. acknowledges the financial support from the European Research Council (ERC) under the European Union's Horizon 2020 research and innovation program (No.803004).

\section{Author contributions}

F.E-A and L.P conceptualized the study. F.E-A. I.C., D.S-F, R.R., A.C-G and L.P. performed experiments and/or analyzed results. F.E-A. and J.V. generated the recombinant proteins used in this study. T.L. and A.C. produced the X. laevis egg extracts. S.V participated in the production of plasma membrane sheets. V.B. designed the automated analysis of images. C.A-A. generated the HT1080 stable cell lines. F.E-A and L.P wrote the manuscript. All authors contributed to the last version of the manuscript. 


\section{Supporting information}

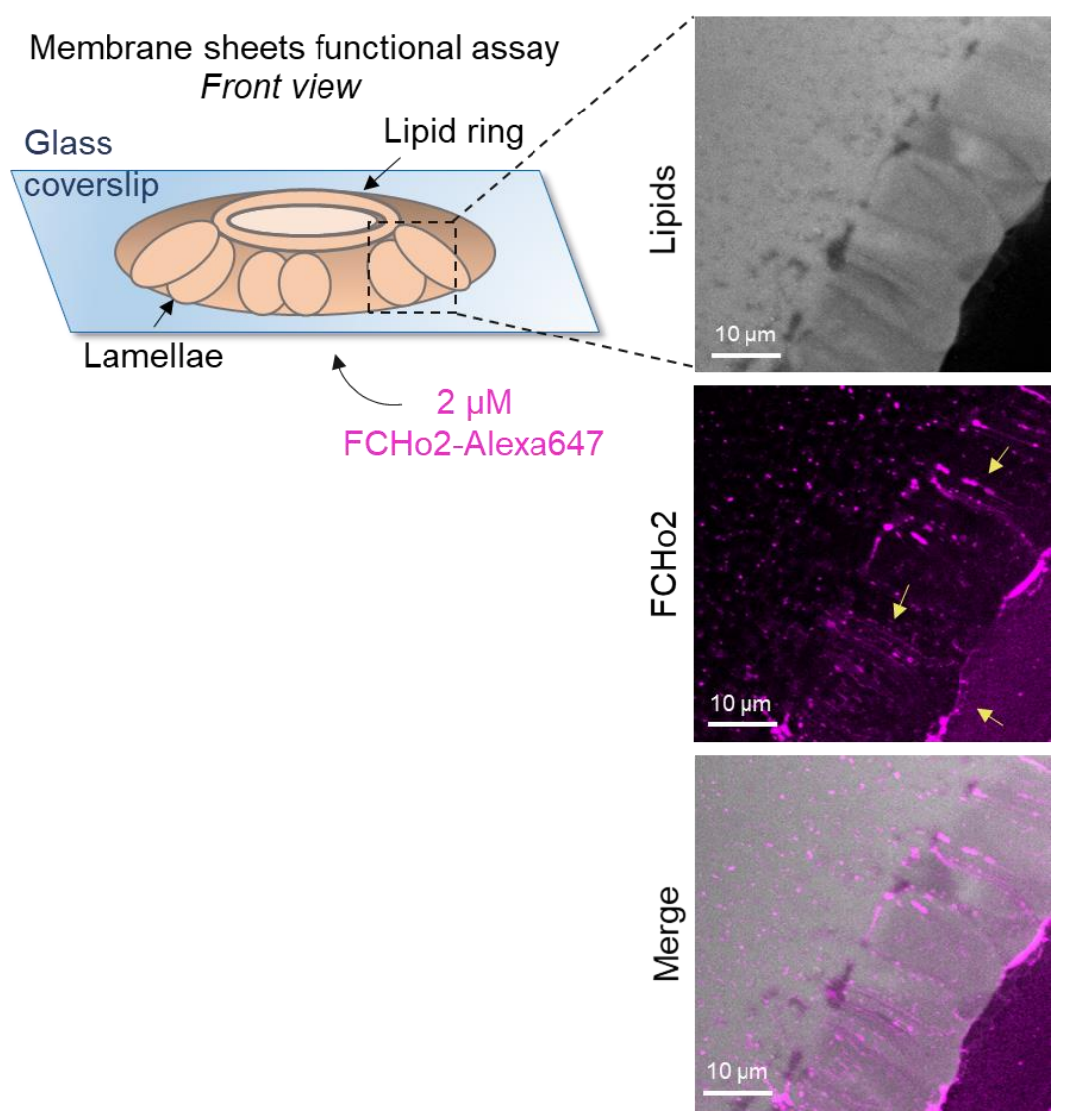

Figure S1. Left, schematic representation of the membrane sheets in vitro assay, as detailed by Itoh et al.(15), to test the functionality of recombinant full-length FCHo2-Alexa647. Membrane sheets were made using brain polar lipid extracts (Avanti). Right, representative still confocal images showing the formation of membrane tubes after addition of $2 \mu \mathrm{M}$ of FCHo2, as indicated by yellow arrows.

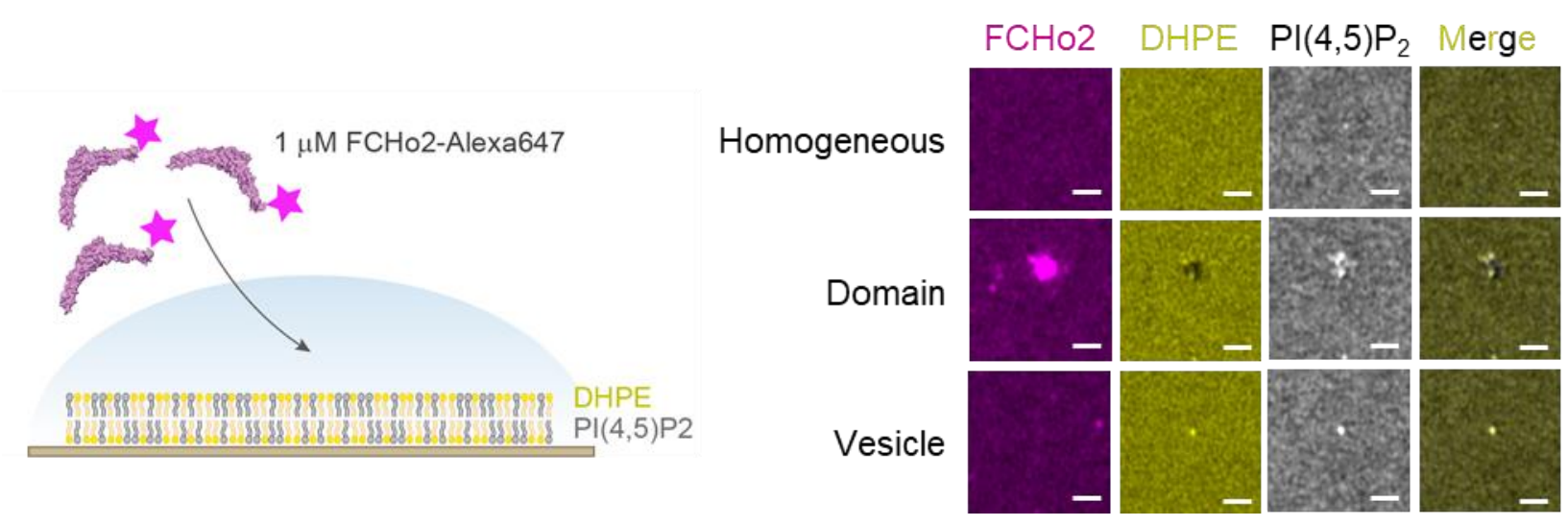

Figure S2. Left, cartoon of the experimental setup to detect the interaction of FCHo2-A647 with PI(4,5) $\mathrm{P}_{2}$-enriched domains. Right, representative airyscan images showing the lipid organizations that were detected under the experimental conditions: (i) an homogenous $\mathrm{PI}(4,5) \mathrm{P}_{2}$ organization, (ii) the formation of lipid domains and (iii) the presence of adsorbed lipid vesicles. Scale bar, $1 \mu \mathrm{m}$. 

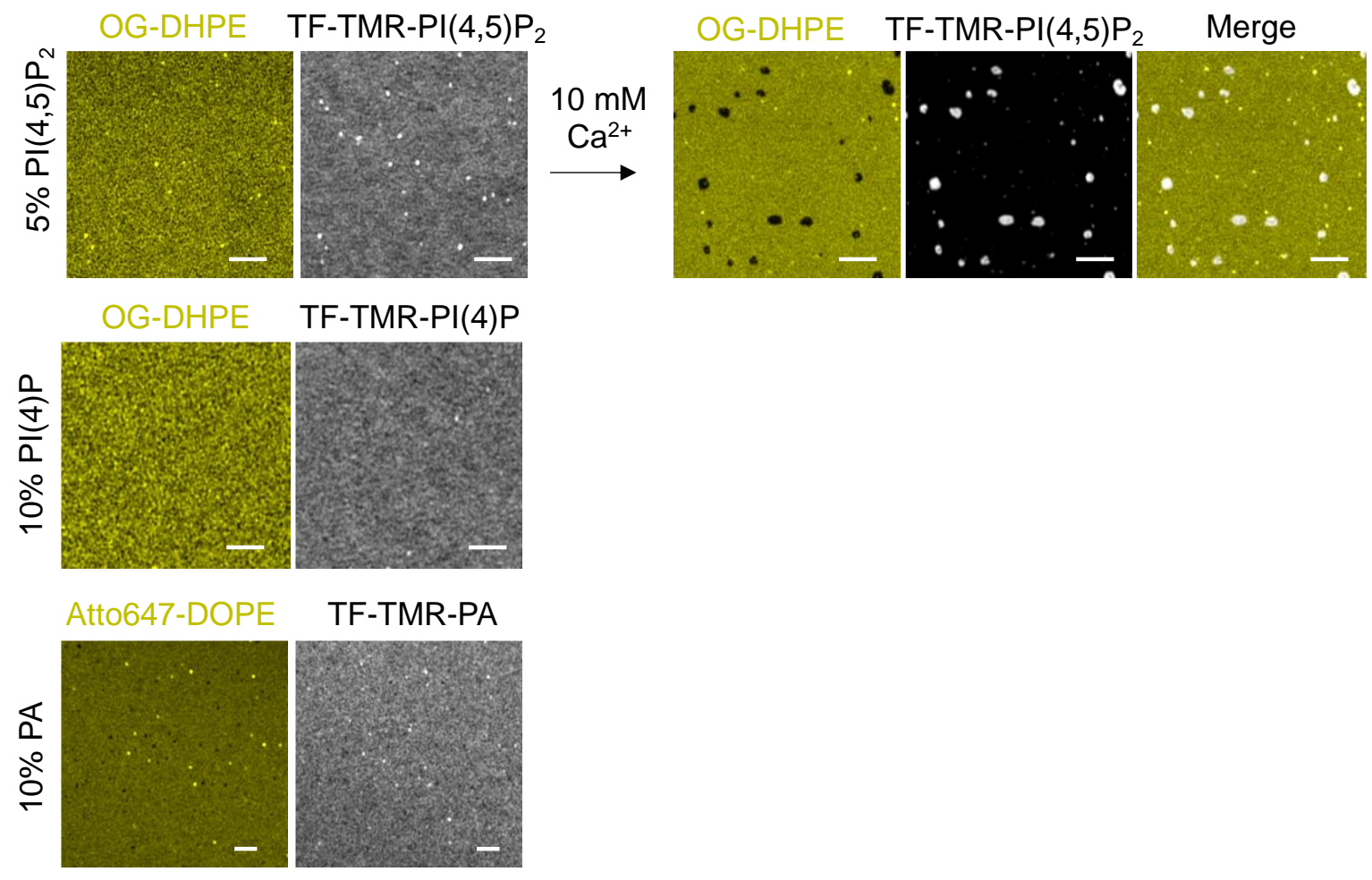

Figure S3. Representative airyscan images showing the lipid organization of different acidic TF-TMR lipid dyes $\left(\mathrm{PI}(4,5) \mathrm{P}_{2}\right.$, $\mathrm{PI}(4) \mathrm{P}$ and phosphatidic acid (PA) in gray) on lipid bilayers containing $20 \%$ mol of total negative charge relative to a neutral lipid dye (OG-DHPE or Atto647-DOPE). Lipid dyes were added to a final $0.2 \%$ mol at the expenses of the non-labeled counterpart. Addition of $10 \mathrm{mM}$ of $\mathrm{Ca}^{2+}$ on lipid bilayers made of $5 \% \mathrm{PI}(4,5) \mathrm{P}_{2}$ and doped with OG-DHPE and TF-TMR$\mathrm{PI}(4,5) \mathrm{P}_{2}$ shows the detection of $\mathrm{PI}(4,5) \mathrm{P}_{2}$-enriched domains on this supported lipid bilayers. Scale bar, $2 \mu \mathrm{m}$.

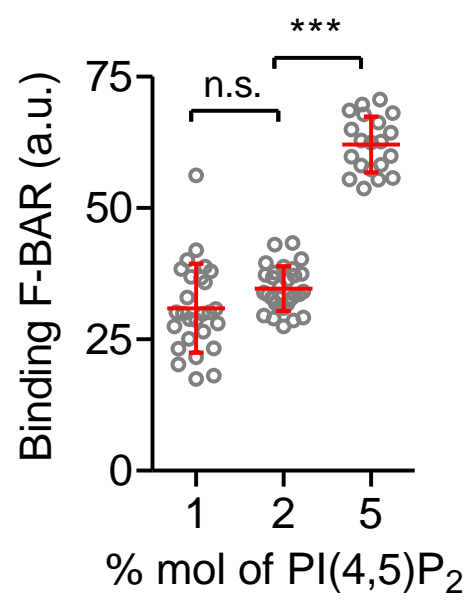

Figure S4. Binding of F-BAR on membranes containing different $\%$ mol of $\mathrm{PI}(4,5) \mathrm{P}_{2}$. Mean \pm s.d. is displayed in red and oneway ANOVA in black. $\mathrm{n}=27, \mathrm{n}=29$, and $\mathrm{n}=20$ for $1 \%, 2 \%$, and $5 \%$ mol of $\mathrm{PI}(4,5) \mathrm{P}_{2}$ respectively. 


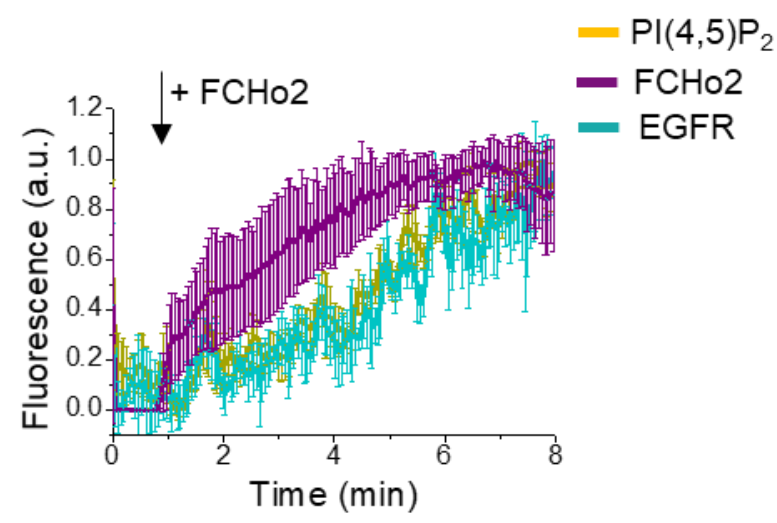

Figure S5. Fluorescence quantification over time of the EGFR (cyan), PI(4,5)P (yellow) upon injection of $1 \mu \mathrm{M}$ of FCHo2Alexa647 (magenta) on EGFR-positive spots on plasma membrane sheets. Each curve represents the mean \pm s.d., $\mathrm{n}=7$.
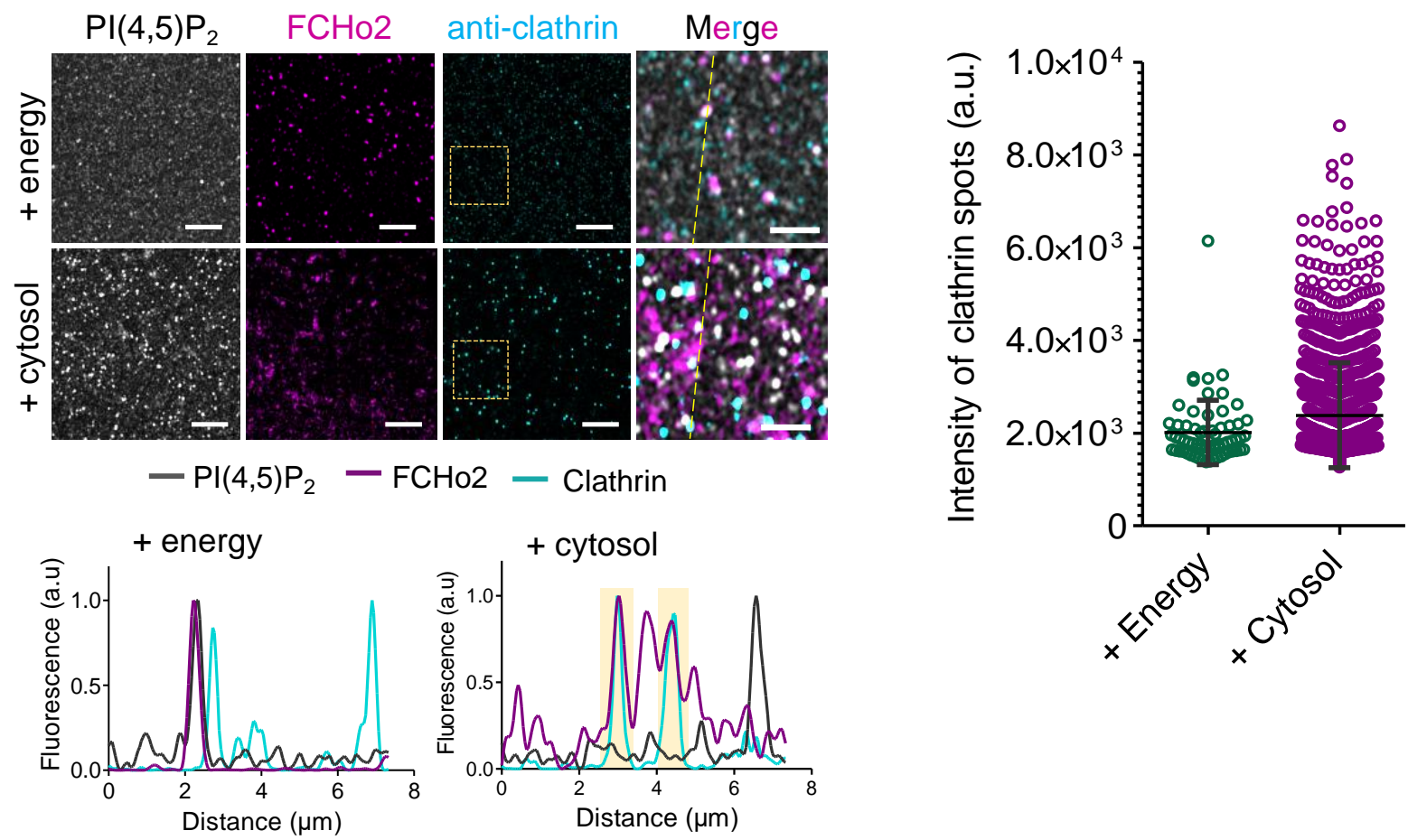

Figure S6. Representative airyscan images of the immunofluorescence assay showing the localization of $\mathrm{PI}(4,5) \mathrm{P}_{2}($ gray), FCHo2 (magenta) and clathrin (cyan, anti-clathrin antibody) on 5\% PI(4,5) $\mathrm{P}_{2}$-containing lipid bilayers incubated with the energy mix alone (+ energy) or with the cytosolic extract and energy mix (+ cytosol). Sale bar, $5 \mu \mathrm{m}$. 